\title{
Zur Lösung des quantenmechanischen Drei-Körper-Problems
}

\author{
H. RUDER
}

Institut für Theoretische Physik der Universität Erlangen-Nürnberg

(Z. Naturforsch. 23 a, 579-596 [1968]; eingegangen am 11. Januar 1968)

\begin{abstract}
For the description of a three-body-system 6 coordinates are introduced which take full advantage of the symmetry properties of the system. The Schrödinger equation in these coordinates is derived. Using rotational and reflection symmetry one obtains a set of $l$ respectively $l+l$ coupled differential equations containing only the 3 coordinates of the triangle formed by the 3 masses. Solutions are given for special potentials and arbitrary $l$. The physical meaning of the differential operators appearing in the equations becomes evident from their application to the solution functions. This leads to a rearrangement of the Hamiltonian in a very transparent form and gives a hint how to get the most effective perturbation expansion. A simple example is worked out. For systems with Coulomb interaction a modification of the method is suggested by physical considerations. The calculation of the ground state energy of the Helium atom shows the rapid convergence of the procedure.
\end{abstract}

\section{A. Koordinatenwahl}

Die in der Natur vorkommenden Systeme aus 3 Teilchen unterscheiden sich durch die Masse der Teilchen und durch die Wechselwirkung zwischen ihnen. Für das folgende nehmen wir an, daß die Wechselwirkung nur eine Funktion der 3 Abstände ist, also keine Spin-Bahn-Kopplung oder ähnliches vorkommt. Wir suchen zunächst Koordinaten, die den allgemeinen physikalischen Symmetrien des Systems angepaßt sind, ohne daß wir dabei auf spezielle Potentiale bzw. Massenverhältnisse Bezug nehmen. Da die meisten Arbeiten über das quantenmechanische 3-Körper-Problem im wesentlichen durch die verwendeten Koordinaten charakterisiert sind, geben wir gleichzeitig einen Uberblick über bisher verwendete Ansätze und diskutieren sie kurz.

\section{Allgemeine Überlegungen}

Da sich die Schwerpunktsbewegung abseparieren läßt, legen wir den Ursprung des raumfesten Koordinatensystems $X Y Z$ in den als ruhend gedachten Schwerpunkt. Zur Beschreibung der Lage der 3 Massenpunkte genügen dann 6 Koordinaten. Für diese gibt es 2 Möglichkeiten.

a) Man beschreibt jeden Massenpunkt unter Mitverwendung des Schwerpunktsatzes vom raumfesten System aus.

b) Man verwendet 3 Koordinaten, um die Gestalt des Dreiecks festzulegen, das von den 3 Massenpunkten gebildet wird, und die restlichen 3 für die Orientierung des Dreiecks im Raum.

Ein Spezialfall von a) wird von $\mathrm{WeIGERT}^{1}$ behandelt. Die von ihm gewählten Koordinaten liefern

1 L. Weigert, Dissertation, Erlangen 1959. einen einfachen Ausdruck für die kinetische Energie, aber die potentielle Energie hängt im allgemeinen von allen 6 Koordinaten ab. Daher kann man für ein beliebiges Potential nur wenig Allgemeines aussagen. Für unser Ziel ist zweifellos b) besser geeignet, denn hierbei wird der physikalische Tatbestand, daß die potentielle Energie nur von der relativen Lage der 3 Massenpunkte abhängt, voll ausgenutzt. Die potentielle Energie ist dann eine Funktion der 3 Koordinaten, die die Gestalt des Dreiecks festlegen. Diese Koordinaten nennen wir innere, die 3 restlichen äußere Koordinaten.

\section{Innere Koordinaten}

Für die Wahl der inneren Koordinaten hat man folgende Möglichkeiten:

a) 3 Längen im Dreieck,

b) 2 Längen und 1 Winkel,

c) 1 Länge und 2 Winkel.

Je nach der Art des vorliegenden Problems wird sich eine der Möglichkeiten als besonders vorteilhaft erweisen.

Im Fall a) ist es naheliegend, für die 3 Längen die gegenseitigen Abstände der 3 Massenpunkte zu wählen, wie es z. B. FLÜGGE und Mitarbeiter ${ }^{2}$ tun. Die potentielle Energie nimmt dann eine einfache Gestalt an, während sich für die kinetische Energie ein recht komplizierter Ausdruck ergibt. Diese Koordinaten zeichnen kein Teilchen aus; sie sind daher besonders der Behandlung von Systemen angepaßt, bei denen die 3 Teilchen entweder alle gleich oder aber alle wirklich verschieden sind.

\footnotetext{
2 H. Diehl, S. FlügGe, U. Schröder, A. Völkel u. A. WeiGUNY, Z. Phys. 162, l [1961].
} 
Für den Fall b) bieten sich mehrere Möglichkeiten an, so zum Beispiel 2 Seiten und der dazwischenliegende Winkel. Jedoch ist eine Möglichkeit, die von Manneback und seinem Schüler Vo-The-Hao ${ }^{3}$, sowie von NÄPfEL und RUdER ${ }^{4}$ verwendet wird, besonders ausgezeichnet. Sie wird in Abb. 1 a erläutert.

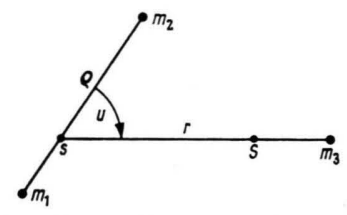

Abb. 1 a. s: Schwerpunkt von $m_{1}$ und $m_{2}$. S: Gesamtschwerpunkt.

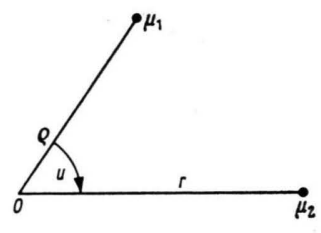

Abb. 1 b. Reduziertes System. 0 : Ursprung des raumfesten Koordinatensystems.
Abb. 1. Koordinaten im 3-Teilchen-System. Variationsbereich von $\varrho, r, u: 0 \leqq \varrho \leqq \infty, 0 \leqq r \leqq \infty, 0 \leqq u \leqq \pi$.

Die Koordinaten $\varrho, r$ und $u$ sollen auch in der vorliegenden Arbeit benutzt werden. Sie haben zwei wesentliche Vorteile:

1. Der Ausdruck für die kinetische Energie des 3-Teilchen-Systems wird gleich dem für ein 2-Teilchen-System mit den Massen

$$
\mu_{1}=\frac{m_{1} m_{2}}{m_{1}+m_{2}} \text { und } \mu_{2}=\frac{\left(m_{1}+m_{2}\right) m_{3}}{m_{1}+m_{2}+m_{3}}
$$

in der Anordnung von Abb. $1 \mathrm{~b}$. Die Umrechnung findet man in ${ }^{4}$. Dieses 2-Teilchen-System nennen wir das reduzierte System, $\mu_{1}$ und $\mu_{2}$ die reduzierten Massen. Wir können nun mit dem einfacheren reduzierten System rechnen, müssen aber beachten, daß alle physikalischen Überlegungen am 3-TeilchenSystem durchzuführen und dann auf das 2-TeilchenSystem zu übertragen sind. So gehen beispielsweise in die potentielle Energie nicht die geometrischen Abstände des reduzierten Systems ein, sondern die wirklichen Abstände

$$
\begin{array}{r}
\varrho, \sqrt{r^{2}+\left(\frac{m_{1}}{m_{1}+m_{2}} \varrho\right)^{2}-2 r\left(\frac{m_{1}}{m_{1}+m_{2}} \varrho\right) \cos u,} \\
\sqrt{r^{2}+\left(\frac{m_{2}}{m_{1}+m_{2}} \varrho\right)^{2}+2 r\left(\frac{m_{2}}{m_{1}+m_{2}} \varrho\right) \cos u} .
\end{array}
$$

2. Die physikalisch interessanten 3-Teilchen-Systeme enthalten meist 2 gleiche Teilchen. Da unsere Koordinaten 2 Teilchen auszeichnen, können wir diese Symmetrie in einfacher Weise verwerten. Das zeigt sich unter anderem daran, daß bei der Vertau-

3 Vo-The-Hao, Dissertation, Louvain 1960.

${ }^{4}$ H. Näpfel u. H. Ruder, Diplomarbeiten, Erlangen 1964 (unveröffentlicht). schung von Teilchen 1 und 2, falls $m_{1}=m_{2}$ ist, nur eine innere Koordinate geändert wird: der Winkel $u$ geht in $\pi-u$ über.

Fall c) dürfte besonders für Probleme geeignet sein, bei denen der Endzustand aus 3 auseinander fliegenden Teilchen besteht, weil dabei asymptotisch das von den 3 Teilchen gebildete Dreieck ähnlich bleibt und sich also nur eine Koordinate, nämlich die Länge, ändert. Zum Fall c) gehören auch die von ZickendRAht ${ }^{5,6}$ verwendeten Koordinaten.

\section{III. Äußere Koordinaten}

Wir müssen jetzt die Orientierung des Dreiecks im Raum angeben. Dazu führen wir ein rechtwinkliges Koordinatensystem $\xi \eta \zeta$ ein, dessen Lage durch die 3 Massenpunkte eindeutig bestimmt ist. Dieses Koordinatensystem nennen wir körpergebunden. Sein Ursprung soll mit dem Ursprung $\mathrm{S}$ des raumfesten Systems zusammenfallen, seine Orientierung gegenüber $X Y Z$ wird durch Eulersche Winkel $\alpha \beta \gamma$ festgelegt, die dann unsere äußeren Koordinaten sind.

Für die Einführung des körpergebundenen Koordinatensystems gibt es verschiedene Möglichkeiten. Eine Achsenrichtung, nämlich die Flächennormale, ist durch das System ausgezeichnet. Die für die beiden restlichen Achsen noch verbleibenden Möglichkeiten unterscheiden sich nur durch eine Drehung um diese Achse. Inwieweit dadurch die Schrödinger-Gleichung beeinflußt wird, wurde in ${ }^{4}$ genau untersucht. Auf Grund dieser Ergebnisse nehmen wir als zweite Richtung die $\varrho$-Richtung. Die Modifikationen, die sich bei Wahl der an sich gleichberechtigten $r$-Richtung ergeben, werden am Schluß diskutiert. In beiden Fällen ist die für die Anwendung wichtige Symmetrie zweier gleicher Teilchen berücksichtigt.

Es gibt jetzt noch mehrere Möglichkeiten, die Achsen mit $\xi, \eta, \zeta \mathrm{zu}$ bezeichnen. Da hierdurch üblicherweise festgelegt wird, wie die Eulerschen Winkel gezählt werden, wirkt sich auch diese Entscheidung in der Schrödinger-Gleichung aus. Es zeigt sich, daß die Wahl der $Q$ - (bzw. $r$-) Richtung als $\zeta$-Achse und damit als körpergebundene Quantisierungsachse zu einer besonders übersichtlichen Schrödinger-Gleichung führt. Die $\eta$-Achse soll in Richtung der Flächennormalen liegen. Die gewählten Koordinaten sind in Abb. 2 dargestellt.

\footnotetext{
5 W. Zickendraht, Proc. Natl. Acad. Sci. U.S. 52, 1565 [1964].

6 W. Zickendraht, Ann. Phys. New York 35, 18 [1965].
} 


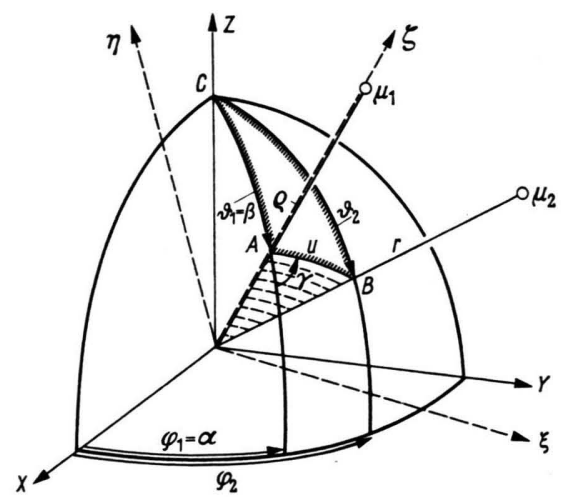

Abb. 2. Innere Koordinaten $\varrho r u$ und äußere Koordinaten $\alpha \beta \gamma$.

$\varrho \vartheta_{1} \varphi_{1}$ und $r \vartheta_{2} \varphi_{2}$ sind die sphärischen Polarkoordinaten für die reduzierten Massen $\mu_{1}$ und $\mu_{2}$ im raumfesten System $X Y Z$. Der Winkel $u$ ist der Polarwinkel von $\mu_{2}$ im körpergebundenen Koordi- natensystem $\xi \eta \zeta$. Der dazugehörende Azimutwinkel ist Null, da $\mu_{2}$ in der $\xi-\zeta$-Ebene liegt. Diese Feststellungen werden dann wichtig, wenn wir die Lösungsfunktionen und Operatoren der SchrödingerGleichung interpretieren.

Verhalten bei Symmetrieoperationen:

1. Spiegelung am Ursprung

Wir nennen den Paritätsoperator $P$, seine Wirkung auf die Koordinaten ist:

$$
\begin{gathered}
\alpha \rightarrow \pi+\alpha, \beta \rightarrow \pi-\beta, \gamma \rightarrow \pi-\gamma, \\
u \rightarrow u, g \rightarrow \varrho, \quad r \rightarrow r .
\end{gathered}
$$

2. Vertauschung von Teilchen 1 und 2

Den Austauschoperator bezeichnen wir mit $E_{12}$; wenn $m_{1}=m_{2}$ ist, bewirkt er folgende Änderung in den Koordinaten:

$$
\begin{gathered}
\alpha \rightarrow \pi+\alpha, \quad \beta \rightarrow \pi-\beta, \quad \gamma \rightarrow 2 \pi-\gamma ; \\
u \rightarrow \pi-u, \quad \varrho \rightarrow \varrho, \quad r \rightarrow r .
\end{gathered}
$$

\section{B. Schrödinger-Gleichung}

Die Schrödinger-Gleichung für unser System in den Koordinaten $\varrho, r, u ; \alpha, \beta, \gamma$ erhalten wir am einfachsten auf dem in ${ }^{7}$ angedeuteten Weg. Es ergibt sich:

$H \Psi=\left(H_{i}+H_{a}+H_{k}\right) \Psi=E \Psi$,

$$
\begin{aligned}
& H_{i}=-\frac{\hbar^{2}}{2 \mu_{1}}\left(\begin{array}{ccc}
\partial^{2} \\
\partial \varrho^{2}
\end{array}+\frac{2}{\varrho} \frac{\partial}{\partial \varrho}\right)-\frac{\hbar^{2}}{2 \mu_{2}}\left(\frac{\partial^{2}}{\partial r^{2}}+\frac{2}{r} \frac{\partial}{\partial r}\right) \\
& -\left(\frac{\hbar^{2}}{2 \mu_{1} \varrho^{2}}+\frac{\hbar^{2}}{2 \mu \mu_{2} r^{2}}\right)\left(\frac{\partial^{2}}{\partial u^{2}}+\cot u \frac{\partial}{\partial u}+\frac{1}{\sin ^{2} u} \frac{\partial^{2}}{\partial \gamma^{2}}\right)+V(\varrho r u) \text {, } \\
& H_{a}=-\frac{\hbar^{2}}{2 \mu_{1} \varrho^{2}}\left[\begin{array}{c}
\partial^{2} \\
\partial \beta^{2}
\end{array}+\cot \beta \underset{\partial \beta}{\partial}+\frac{1}{\sin ^{2} \beta}\left(\begin{array}{c}
\partial^{2} \\
\partial \gamma^{2}
\end{array}-2 \cos \beta \frac{\partial^{2}}{\partial \gamma \partial \alpha}+\frac{\partial^{2}}{\partial a^{2}}\right)-2 \frac{\partial^{2}}{\partial \gamma^{2}}\right] \\
& H_{k}=\frac{1}{2 \mu_{1} \varrho^{2}}\left[\frac{\hbar}{i} e^{-i \gamma}\left(\cot \beta \frac{\partial}{\partial \gamma}+i \frac{\partial}{\partial \beta}-\frac{1}{\sin \beta} \frac{\partial}{\partial x}\right)\left(\cot u \frac{\hbar}{i} \frac{\partial}{\partial \gamma}+\hbar \frac{\partial}{\partial u}\right)\right. \\
& \left.+\frac{\hbar}{i} e^{i \gamma}\left(\cot \beta \frac{\partial}{\partial \gamma}-i \frac{\partial}{\partial \beta}-\frac{1}{\sin \beta} \frac{\partial}{\partial \alpha}\right)\left(\cot u \stackrel{\hbar}{i} \frac{\partial}{\partial \gamma}-\hbar \frac{\partial}{\partial u}\right)\right] .
\end{aligned}
$$

Für $m_{1}=\infty, m_{2}=m_{3}=$ Elektronenmasse geht (3) in die Schrödinger-Gleichung von HylleraAs ${ }^{8}$ für 2 Elektronen im Zentralfeld über. Bei dieser Zuordnung hat man allerdings nicht mehr das einfache Verhalten bei Vertauschung der beiden Elektronen.

Für das Folgende erweist es sich als zweckmäßig, den Gesamtdrehimpuls $\mathbf{L}$ auf die Achsen des körpergebundenen Koordinatensystems zu zerlegen. Diese Komponenten nennen wir kurz körpergebundene Komponenten. Während in der klassischen Physik ein Drehimpulsvektor immer ohne Schwierigkeiten auch auf mitbewegte Achsen zerlegt werden kann, ist beim quantenmechanischen Drehimpulsoperator Vorsicht geboten, denn die körpergebundenen Komponenten zeigen hier ein anderes Verhalten als die raumfesten. Die auftretenden Probleme wurden in ${ }^{7}$ erörtert. Wir geben an dieser Stelle nur die Ausdrücke an, die wir später brauchen.

Die körpergebundenen Komponenten von $\mathbf{L}$ sind in unserem Fall:

$$
\begin{aligned}
& L_{\xi}=\frac{\hbar}{i}\left(\sin \gamma \frac{\partial}{\partial \beta}-\frac{\cos \gamma}{\sin \beta} \frac{\partial}{\partial \alpha}+\cot \beta \cos \gamma \frac{\partial}{\partial \gamma}\right), \\
& L_{\eta}=\frac{\hbar}{i}\left(\cos \gamma \frac{\partial}{\partial \beta}+\frac{\sin \gamma}{\sin \beta} \frac{\partial}{\partial \alpha}-\cot \beta \sin \gamma \frac{\partial}{\partial \gamma}\right), \quad L_{\zeta}=\frac{\hbar}{i} \frac{\partial}{\partial \gamma} .
\end{aligned}
$$

Sie genügen den „anomalen“ Vertauschungsrelationen: $L_{\xi} L_{\eta}-L_{\eta} L_{\xi}=(\hbar / i) \mathrm{L}_{\zeta}$ und zyklisch.

7 H. Näpfel, H. Ruder u. H. Volz, Z. Naturforsch. 23 a, $199 \quad{ }^{8}$ E. A. Hylleraas, Z. Phys. 48, 469 [1928]. [1968]. 
Wir definieren noch folgende Operatoren:

$$
\begin{aligned}
& L_{+}=L_{\xi}+i L_{\eta}=\frac{\hbar}{i} e^{-i \gamma}\left(\cot \beta \frac{\partial}{\partial \gamma}+i \frac{\partial}{\partial \beta}-\frac{1}{\sin \beta} \frac{\partial}{\partial \alpha}\right), \\
& L_{-}=L_{\xi}-i L_{\eta}=\frac{\hbar}{i} e^{i \gamma}\left(\cot \beta \frac{\partial}{\partial \gamma}-i \frac{\partial}{\partial \beta}-\frac{1}{\sin \beta} \frac{\partial}{\partial \alpha}\right), \\
& L^{2}=L_{\xi}^{2}+L_{\eta}^{2}+L_{\zeta}^{2}=-\hbar^{2}\left[\frac{\partial^{2}}{\partial \beta^{2}}+\cot \beta \frac{\partial}{\partial \beta}+\frac{1}{\sin ^{2} \beta}\left(\frac{\partial^{2}}{\partial \gamma^{2}}-2 \cos \beta \frac{\partial^{2}}{\partial \gamma \partial \alpha}+\frac{\partial^{2}}{\partial \alpha^{2}}\right)\right] .
\end{aligned}
$$

Mit diesen Operatoren läßt sich die Schrödinger-Gleichung (3) wie folgt schreiben:

$$
\left\{H_{i}+\frac{1}{2 \mu_{1} \varrho^{2}}\left(L^{2}-2 L_{\zeta}^{2}\right)+\frac{1}{2 \mu_{1} \varrho^{2}}\left[L_{+}\left(\cot u L_{\zeta}+\hbar \frac{\partial}{\partial u}\right)+L_{-}\left(\cot u L_{\zeta}-\hbar \frac{\partial}{\partial u}\right)\right]\right\} \Psi=E \Psi^{\prime} .
$$

Eine ähnliche Zusammenfassung wurde von Manneвack angegeben (mündliche Mitteilung).

\section{Invarianzen des Hamilton-Operators}

\section{Drehinvarianz}

Da die potentielle Energie nur von den inneren Koordinaten abhängt, ist der Hamilton-Operator drehinvariant. Die Eigenfunktionen lassen sich dann nach den Darstellungskoeffizienten der Drehgruppe entwickeln. Im Laufe dieser Arbeit hat es sich als zweckmäßig erwiesen, mit den Darstellungskoeffizienten $D_{m n}^{l^{*}}(\alpha \beta \gamma)$ von $\operatorname{Rose}^{9}$ zu rechnen; man vermeidet dadurch unter anderem Faktoren wie $(-1)^{m-n}$. Wir notieren einige Eigenschaften dieser $D_{m n}^{l^{*}}(\alpha \beta \gamma)$ :

$$
\begin{aligned}
& D_{m n}^{l^{*}}(\alpha \beta \gamma)=e^{i m a} d_{m n}^{l}(\beta) e^{i n \gamma}, \\
& \int_{0}^{2 \pi} \int_{0}^{\pi} \int_{0}^{2 \pi} D_{m n}^{l}(\alpha \beta \gamma) D_{m^{\prime} n^{\prime}}^{l^{\prime *}}(\alpha \beta \gamma) \mathrm{d} \alpha \sin \beta \mathrm{d} \beta \mathrm{d} \gamma=\frac{8 \pi^{2}}{2 l+1} \delta_{m m^{\prime}} \delta_{n n^{\prime}} \delta_{l l^{\prime}}, \\
& L^{2} D_{m n}^{l^{*}}=\hbar^{2} l(l+1) D_{m n}^{l^{*}}, \quad L_{\zeta} D_{m n}^{l^{*}}=\hbar n D_{m n}^{l^{*}} ;
\end{aligned}
$$

$l$ ist die Quantenzahl des Gesamtdrehimpulses, $m$ die Projektion auf die raumfeste $Z$-Achse und $n$ die Projektion auf die körpergebundene $\zeta$-Achse.

$$
L_{+} D_{m n}^{l^{*}}=\hbar \sqrt{(l+n)(l-n+1)} D_{m n-1}^{l^{*}}, \quad L_{-} D_{m n}^{l^{*}}=\hbar \sqrt{(l-n)(l+n+1)} D_{m n+1}^{l^{*}} .
$$

Dieser gegenüber den raumfesten Operatoren umgekehrte Schraubsinn von $L_{+}$und $L_{-}$folgt aus den anomalen Vertauschungsrelationen.

Die Drehinvarianz des Hamilton-Operators liefert also folgende Gestalt der Eigenfunktionen für einen Zustand mit gegebenem $l$ und $m$ :

$$
\Psi_{m}^{l}(\varrho r u, \alpha \beta \gamma)=\sum_{n=-l}^{n=+l} \Phi_{m n}^{l}(\varrho r u) D_{m n}^{l^{*}}(\alpha \beta \gamma) .
$$

Die Projektionsquantenzahl $n$ nimmt im allgemeinen alle Werte von $-l$ bis $+l$ an, da unser HamiltonOperator einen Teil, nämlich $H_{k}$, enthält, der gegenüber Drehungen um die $\zeta$-Achse nicht invariant ist. Zur vollständigen Lösung der Schrödinger-Gleichung müssen wir jetzt noch die $2 l+1$ unbekannten Funktionen $\Phi_{m n}(\varrho r u)$ bestimmen.

\section{Parität}

Der Hamilton-Operator unseres Systems ist invariant gegen Spiegelung am Ursprung. Die Eigenfunktionen besitzen daher Parität: $P \Psi= \pm \Psi$. Von (1) wissen wir, wie der Paritätsoperator $P$ auf die Koordinaten wirkt; damit erhalten wir:

$$
P D_{m n}^{l^{*}}(\alpha \beta \gamma)=D_{m n}^{l^{*}}(\pi+\alpha, \pi-\beta, \pi-\gamma)=(-1)^{l+n} D_{m-n}^{l^{*}}(\alpha \beta \gamma) .
$$

Mit dieser Überlegung läßt sich unser Lösungsansatz weiter einschränken. Wir bilden dazu Funktionen mit bestimmter Parität:

$$
\begin{array}{ll}
\mathcal{L}_{m n}^{l \pm}(\alpha \beta \gamma)=\frac{1}{\sqrt{2}}\left\{D_{m n}^{l^{*}}(\alpha \beta \gamma) \pm(-1)^{l+n} D_{m-n}^{l^{*}}(\alpha \beta \gamma)\right\}, & n \neq 0, \\
\mathcal{L}_{m 0}^{l \pm}(\alpha \beta \gamma)=\frac{1}{2}\left\{D_{m 0}^{l^{*}}(\alpha \beta \gamma) \pm(-1)^{l} D_{m 0}^{l^{*}}(\alpha \beta \gamma)\right\}, & n=0 .
\end{array}
$$

๑ M. E. Rose, Elementary Theory of Angular Momentum, John Wiley \& Sons, New York 1966. 
Die Funktionen $\mathcal{L}_{m n}^{l+}$ haben positive, $\mathcal{L}_{m n}^{l-}$ negative Parität: $P \mathcal{L}_{m n}^{l \pm}= \pm \mathcal{L}_{m n}^{l \pm}, \mathcal{L}_{m 0}^{l+} \equiv 0$ für $l$ ungerade, $\mathcal{L}_{m 0}^{l-} \equiv 0$ für $l$ gerade.

Der Faktor $1 / \sqrt{2}$ bzw. $1 / 2$ dient nur dazu, daß die $\mathcal{L}_{m n}^{l_{m}^{ \pm}}$dieselbe Normierung besitzen wie die $D_{m n}^{l^{*}}$. Unsere Lösungsfunktionen mit definierter Parität werden dann:

$$
\Psi_{m}^{l \pm}=\sum_{n=0, n-1}^{n=l} \Phi_{m n}^{l \pm}(\varrho r u) \mathcal{L}_{m n}^{l \pm}(\alpha \beta \gamma) ; \quad P \Psi_{m}^{l_{ \pm}}= \pm \Psi_{m}^{l \pm}
$$

Auf Grund dieser Paritätsüberlegung reduziert sich für einen Zustand mit Drehimpuls $l$ und gegebener Parität die Zahl der unbekannten Funktionen $\Phi_{m n}^{l \pm}$ auf $l$ bzw. $l+1$.

\section{Teilchenvertauschung}

Wir betrachten den Fall, daß in unserem System 2 Teilchen gleich sind. Der Hamilton-Operator ist dann invariant gegen Vertauschung der beiden Teilchen, und die Wellenfunktion verhält sich entweder symmetrisch oder antisymmetrisch bei dieser Operation. Die beiden gleichen Teilchen werden bei unserer Wahl der Koordinaten die Teilchen 1 und 2. Wie sich bei ihrer Vertauschung die Koordinaten ändern, haben wir in (2) angegeben. Damit läßt sich die Wirkung des Austauschoperators $E_{12}$ auf unseren Lösungsansatz berechnen:

$$
\begin{aligned}
& E_{12} D_{m n}^{l^{*}}(\alpha \beta \gamma)=D_{m n}^{l^{*}}(\pi+\alpha, \pi-\beta, 2 \pi-\gamma)=(-1)^{l} D_{m-n}^{l^{*}}(\alpha \beta \gamma), \\
& E_{12} \mathcal{L}_{m n}^{l_{ \pm}}(\alpha \beta \gamma)= \pm(-1)^{n} \mathcal{L}_{m n}^{l_{ \pm}}(\alpha \beta \gamma) \text {, } \\
& E_{12} \Psi_{m}^{l_{ \pm}}=E_{12} \sum_{n} \Phi_{m n}^{l \pm} \mathcal{L}_{m n}^{l \pm}=\sum_{n}\left(E_{12} \Phi_{m n}^{l \pm}\right) \cdot\left(E_{12} \mathcal{L}_{m n}^{l \pm}\right)= \pm \sum_{n}\left(E_{12} \Phi_{m n}^{l \pm}\right) \cdot(-1)^{n} \mathcal{L}_{m n}^{l \pm}=( \pm) \Psi_{m}^{l \pm} \\
& \rightarrow E_{12} \Phi_{m n}^{l \pm}(\varrho r u)=\Phi_{m n}^{l \pm}(\varrho r \pi-u)= \pm(-1)^{n} \Phi_{m n}^{l \pm}(\varrho r u) .
\end{aligned}
$$

Wir führen die 4 verschiedenen Fälle einzeln auf:

$$
\begin{aligned}
& E_{12} \Psi_{m}^{l+}=+\Psi_{m}^{l+} \rightarrow E_{12} \Phi_{m n}^{l+}=+(-1)^{n} \Phi_{m n}^{l+}, \\
& E_{12} \Psi_{m}^{l+}=-\Psi_{m}^{l+} \rightarrow E_{12} \Phi_{m n}^{l+}=-(-1)^{n} \Phi_{m n}^{l+}, \\
& E_{12} \Psi_{m}^{l-}=+\Psi_{m}^{l-} \rightarrow E_{12} \Phi_{m n}^{l-}=-(-1)^{n} \Phi_{m n}^{l-}, \\
& E_{12} \Psi_{m}^{l-}=-\Psi_{m}^{l-} \rightarrow E_{12} \Phi_{m n}^{l-}=+(-1)^{n} \Phi_{m n}^{l-} .
\end{aligned}
$$

Diese Überlegungen verringern die Anzahl der Funktionen $\Phi_{m n}^{l \pm}$, die für einen Zustand mit gegebenen Quantenzahlen zu bestimmen sind, nicht weiter, wie dies bei der Parität der Fall war, sondern liefern eine Aussage über die Funktionen $\Phi_{m n}^{l \pm}$ selbst. Wir werden deshalb die Lösungstheorie für die äußeren Koordinaten ohne Spezialisierung auf 2 gleiche Teilchen durchführen und erst im Laufe der Lösungstheorie für die inneren Koordinaten wieder darauf zurückkommen.

\section{Lösungstheorie für die äußeren Koordinaten}

Wir müssen nun den Hamilton-Operator auf unseren Lösungsansatz

$$
\Psi_{m}^{l_{ \pm}}(\varrho r u, \alpha \beta \gamma)=\sum_{n} \Phi_{m n}^{l \pm}(\varrho r u) \mathcal{L}_{m n}^{l_{ \pm}}(\alpha \beta \gamma)
$$

wirken lassen und die $\mathcal{L}_{m n}^{l \pm}(\alpha \beta \gamma)$ abseparieren, damit wir Bestimmungsgleichungen für die unbekannten Funktionen $\Phi_{m n}^{l \pm}(\varrho r u)$ erhalten. Dazu untersuchen wir zunächst, wie die einzelnen in der SchrödingerGleichung vorkommenden Operatoren auf die Funktionen $\mathcal{L}_{m n}^{l \pm}(\alpha \beta \gamma)$ wirken.

$$
\begin{aligned}
& L^{2} \mathcal{L}_{m n}^{l \pm}=\hbar^{2} l(l+1) \mathcal{L}_{m n}^{l \pm}, \quad L_{\zeta}^{2} \mathcal{L}_{m n}^{l \pm}=\hbar^{2} n^{2} \mathcal{L}_{m n}^{l \pm}, \\
& H_{i} \mathcal{L}_{m n}^{l \pm}=\left\{-\frac{\hbar^{2}}{2 \mu_{1}}\left(\frac{\partial^{2}}{\partial \varrho^{2}}+\frac{2}{\varrho} \frac{\partial}{\partial \varrho}\right)-\frac{\hbar^{2}}{2 \mu_{2}}\left(\frac{\partial^{2}}{\partial r^{2}}+\frac{2}{r} \frac{\partial}{\partial r}\right)\right. \\
& \left.-\left(\frac{\hbar^{2}}{2 \mu_{1} \varrho^{2}}+\frac{\hbar^{2}}{2 \mu_{2} r^{2}}\right)\left(\frac{\partial^{2}}{\partial u^{2}}+\cot u \frac{\partial}{\partial u}-\frac{n^{2}}{\sin ^{2} u}\right)+V(\varrho r u)\right\} \mathcal{L}_{m n}^{l \pm} \underset{\text { Def. }}{\bar{~}} H_{i n} \mathcal{L}_{m n}^{l \pm}, \\
& H_{a} \mathcal{L}_{m n}^{l \pm}=\frac{\hbar^{2}}{2 \mu_{1} \varrho^{2}}\left\{l(l+1)-2 n^{2}\right\} \mathcal{L}_{m n}^{l \pm}, \quad H_{i n}+\frac{\hbar^{2}}{2 \mu_{1} \varrho^{2}}\left\{l(l+1)-2 n^{2}\right\} \underset{\text { Def. }}{\overline{=}} H_{i n}^{l}, \\
& H_{k} \mathcal{L}_{m n}^{l \pm}=\frac{\hbar^{2}}{2 \mu_{1} \varrho^{2}}\{\sqrt{(l-n)(l+n+1})\left(n \cot u-\frac{\partial}{\partial u}\right) \mathcal{L}_{m n+1}^{l \pm} \\
& \left.+\sqrt{(l+n)(l-n+1)}\left(n \cot u+\frac{\partial}{\partial u}\right) \mathcal{L}_{m n-1}^{l_{ \pm}}\right\} \\
& =b_{n+1}^{l} \mathcal{L}_{m n+1}^{l_{ \pm}}-b_{-(n-1)}^{l} \mathcal{L}_{m n-1}^{l_{ \pm}} ; \quad n \neq 0, n \neq 1, n \neq l
\end{aligned}
$$


mit

$$
b_{n}^{l}=\frac{\hbar^{2}}{2 \mu_{1} \varrho^{2}} \sqrt{(l+n)(l-n+1)}\left((n-1) \cot u-\frac{\partial}{\partial u}\right) .
$$

Die Operatoren $H_{i}$ und $H_{a}$ lassen $n$ unverändert, während $H_{k}$ von $n$ auf $n+1$ und $n-1$ schraubt. An dieser Stelle macht sich bemerkbar, daß $H_{i}$ und $H_{a}$ gegenüber Drehungen um die $\zeta$-Achse invariant sind, $H_{k}$ dagegen nicht.

Das Verhalten für $n=0, n=1$ und $n=l$ wird getrennt untersucht:

$$
\begin{aligned}
& l \text { gerade: }\left\{\begin{array}{lll}
n=0: & \mathcal{L}_{m 0}^{l-} \equiv 0, & H_{k} \mathcal{L}_{m 0}^{l+}=\sqrt{2} b_{1}^{l} \mathcal{L}_{m 1}^{l+}, \\
n=1: & H_{k} \mathcal{L}_{m 1}^{l-}=b_{2}^{l} \mathcal{L}_{m 2}^{l-}, & H_{k} \mathcal{L}_{m 1}^{l+}=b_{2}^{l} \mathcal{L}_{m 2}^{l+}-\sqrt{2} b_{-0}^{l} \mathcal{L}_{m 0}^{l+}
\end{array} ;\right. \\
& l \text { ungerade: }\left\{\begin{array}{lll}
n=0: & \mathcal{L}_{m 0}^{l+} \equiv 0, & H_{k} \mathcal{L}_{m 0}^{l-}=\sqrt{2} b_{1}^{l} \mathcal{L}_{m 1}^{l-}, \\
n=1: & H_{k} \mathcal{L}_{m 1}^{l+}=b_{2}^{l} \mathcal{L}_{m 2}^{l+}, & H_{k} \mathcal{L}_{m 1}^{l-}=b_{2}^{l} \mathcal{L}_{m 2}^{l-}-\sqrt{2} b_{-0}^{l} \mathcal{L}_{m 0}^{l-} ;
\end{array}\right. \\
& \text { für alle } l: \quad n=l: \quad H_{k} \mathcal{L}_{m l}^{l \pm}=-b_{-(l-1)}^{l} \mathcal{L}_{m l}^{l \pm}-1 .
\end{aligned}
$$

Wir wenden jetzt den Hamilton-Operator auf unsere Lösungsfunktion an, und zwar zunächst ohne Berücksichtigung der Sonderfälle $n=0, n=1$ und $n=l$ :

$$
\begin{aligned}
(H-E) \Psi_{m}^{l_{ \pm}} & =\left(H_{i}+H_{a}+H_{k}-E\right) \sum_{n} \Phi_{m n}^{l_{ \pm}} \mathcal{L}_{m n}^{l_{ \pm}} \\
& =\sum_{n}\left\{\left(H_{i n}^{l}-E\right) \Phi_{m n}^{l_{ \pm}} \mathcal{L}_{m n}^{l \pm}+b_{n+1}^{l} \Phi_{m n}^{l_{ \pm}} \mathcal{L}_{m n+1}^{l \pm}-b_{-(n-1)}^{l} \Phi_{m n}^{l_{ \pm}} \mathcal{L}_{m n-1}^{l_{ \pm}}\right\} \\
& =\sum\left\{\left(H_{i n}^{l}-E\right) \Phi_{m n}^{l_{ \pm}}+b_{n}^{l} \Phi_{m n-1}^{l_{ \pm}^{ \pm}}-b_{-n}^{l} \Phi_{m n+1}^{l_{ \pm}}\right\} \mathcal{L}_{m n}^{l_{ \pm}}=0 .
\end{aligned}
$$

Die Funktionen $\mathcal{L}_{m n}^{l \pm}$ sind linear unabhängig; es muß daher jede Klammer für sich verschwinden. Zusammen mit den bereits untersuchten Sonderfällen $n=0, n=1$ und $n=l$ erhalten wir so zu jeder Parität und jedem Drehimpuls ein System von gekoppelten Differentialgleichungen für die Funktionen $\Phi_{m n}^{l_{ \pm}}$. Zur besseren Übersicht schreiben wir die auftretenden Fälle ausführlich hin.

\section{Positive Parität}

$\underline{l=0}: \quad\left(H_{i 0}^{0}-E\right) \Phi_{m 0}^{0+}=0 ;$

$\overline{l=1:} \quad\left(H_{i 1}^{1}-E\right) \Phi_{m 1}^{1+}=0 ;$

$\underline{l=2:} \quad\left(H_{i 0}^{2}-E\right) \Phi_{m 0}^{2+}-\sqrt{2} b_{-0}^{2} \Phi_{m 1}^{2+} \quad=0$

$$
\begin{array}{r}
\sqrt{2} b_{1}^{2} \Phi_{m 0}^{2+}+\left(H_{\imath 1}^{2}-E\right) \Phi_{m 1}^{2+}-b_{-1}^{2} \Phi_{m 2}^{2+}=0 \\
b_{2}^{2} \Phi_{m 1}^{2+}+\left(H_{i 2}^{2}-E\right) \Phi_{m 2}^{2+}=0
\end{array}
$$

\section{l ungerade}

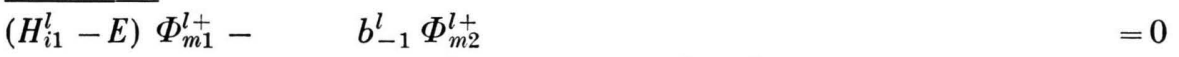

$$
\begin{aligned}
& b_{2}^{l} \Phi_{m 1}^{l+}+\left(H_{i 2}^{l}-E\right) \Phi_{m 2}^{l+}-\quad b_{-2}^{l} \Phi_{m 3}^{l+} \quad=0 \\
& b_{n}^{l} \Phi_{m n-1}^{l+}+\left(H_{i n}^{l}-E\right) \Phi_{m n}^{l+}-\quad b_{-n}^{l} \Phi_{m n+1}^{l+}=0 \\
& \overline{b_{l}^{l}} \Phi_{m l-1}^{l+}+\left(H_{i l}^{l}-\bar{E}\right) \Phi_{m l}^{l+}=0
\end{aligned}
$$

$\underline{l \text { gerade }}$

$$
\begin{aligned}
& \overline{\left(H_{i 0}^{l}-E\right)} \Phi_{m 0}^{l+} \quad-\sqrt{2} b_{-0}^{l} \Phi_{m 1}^{l+} \quad=0 \\
& \sqrt{2} b_{1}^{l} \Phi_{m 0}^{l+}+\left(H_{i 1}^{l}-E\right) \Phi_{m 1}^{l+}-\quad b_{-1}^{l} \Phi_{m 2}^{l+} \quad=0 \\
& b_{2}^{l} \Phi_{m 1}^{l+}+\left(H_{i 2}^{l}-E\right) \Phi_{m 2}^{l+}-\quad b_{-2}^{l} \Phi_{m 3}^{l+} \quad=0 \\
& -\quad-\overline{b_{n}^{l} \Phi_{m n-1}^{l+}+\left(\bar{H}_{i n}^{l}-E\right) \bar{\Phi}_{m n}^{l+}-\quad-\quad b_{-n}^{l} \Phi_{m n+1}^{l+}=0} \\
& \overline{b_{l}^{l}} \Phi_{m l-1}^{l+}+\overline{\left(H_{i l}^{l}-E\right)} \Phi_{m l}^{l+}=0
\end{aligned}
$$


Negative Parität

$\underline{l=0}$ existiert nicht. Der Grundzustand hat immer positive Parität.
$\underline{l=1}:$
$\left(H_{i 0}^{1}-E\right) \Phi_{m 0}^{1-}-$
$\sqrt{2} b_{-0}^{1} \Phi_{m 1}^{1-}=0$,
$\sqrt{2} b_{1}^{1} \Phi_{m 0}^{1-}+\left(H_{i 1}^{1}-E\right) \Phi_{m 1}^{1-}=0 ;$
$\underline{l=2}:$
$\left(H_{i 1}^{2}-E\right) \Phi_{m 1}^{2-}-$
$b_{-1}^{2} \Phi_{m 2}^{2-}=0$,
$b_{2}^{2} \Phi_{m 1}^{2-\overline{1}}+\left(H_{i 2}^{2}-E\right) \Phi_{m \overline{2}}^{2-}=0 ;$

$l$ ungerade bei negativer Parität entspricht dem Fall $l$ gerade bei positiver Parität und umgekehrt.

\section{E. Lösungstheorie für die inneren Koordinaten}

Während sich die Lösungstheorie für die äußeren Koordinaten ohne Annahme über die Form der potentiellen Energie entwickeln ließ, ist das für die inneren Koordinaten nicht mehr möglich. Die Funktionen $\Phi_{m n}^{l \pm}(\varrho r u)$ können ohne Kenntnis des Potentials nicht näher bestimmt werden. Eine exakte Lösung mit bekannten Funktionen wird nur in speziellen Fällen möglich sein; im übrigen wird man numerisch oder mit Störungsrechnung vorgehen müssen. Wir suchen zunächst solche Spezialfälle und diskutieren die Lösungen. Dazu wählen wir einen Weg, der uns ohne viel Rechnung zum Ziel führt.

\section{Spezielle Lösung im raumfesten Koordinaten- system}

Wir erinnern uns an die in Abschnitt A erwähnte Möglichkeit, die 2 reduzierten Massen im raumfesten $X Y Z$-System mit den sphärischen Polarkoordinaten $\varrho, \vartheta_{1}, \varphi_{1}$ und $r, \vartheta_{2}, \varphi_{2}$ zu beschreiben. In diesen Koordinaten können wir den Hamilton-Operator sofort angeben:

$$
\begin{aligned}
& H\left(\varrho \vartheta_{1} \varphi_{1}, r \vartheta_{2} \varphi_{2}\right)= \\
& \quad-\frac{\hbar^{2}}{2 \mu_{1}} \Delta\left(\varrho \vartheta_{1} \varphi_{1}\right)-\frac{\hbar^{2}}{2 \mu_{2}} \Delta\left(r \vartheta_{2} \varphi_{2}\right) \\
& \quad+V\left(\varrho \vartheta_{1} \varphi_{1}, r \vartheta_{2} \varphi_{2}\right)
\end{aligned}
$$

Dabei bedeutet $\Delta\left(\varrho \vartheta_{1} \varphi_{1}\right)$ bzw. $\Delta\left(r \vartheta_{2} \varphi_{2}\right)$ den Laplace-Operator in den entsprechenden Koordinaten.

Wir betrachten jetzt den Fall, daß zwischen den beiden reduzierten Massen keine Wechselwirkung vorhanden ist: die potentielle Energie hat dann die Form

$$
V\left(\varrho \vartheta_{1} \varphi_{1}, r \vartheta_{2} \varphi_{2}\right)=V_{1}\left(\varrho \vartheta_{1} \varphi_{1}\right)+V_{2}\left(r \vartheta_{2} \varphi_{2}\right),
$$

und der Hamilton-Operator ist separierbar in

$$
H\left(\varrho \vartheta_{1} \varphi_{1}, r \vartheta_{2} \varphi_{2}\right)=H_{1}\left(\varrho \vartheta_{1} \varphi_{1}\right)+H_{2}\left(r \vartheta_{2} \varphi_{2}\right) \text {. }
$$

Berücksichtigen wir noch, daß die potentielle Energie bei unseren 3-Teilchen-Systemen nur von den inneren Koordinaten $\varrho, r, u$ abhängt, so erhalten wir die Bedingung:

$$
V_{1}\left(\varrho \vartheta_{1} \varphi_{1}\right)+V_{2}\left(r \vartheta_{2} \varphi_{2}\right)=V(\varrho r u) .
$$

Sie ist nur dann erfüllt, wenn in der potentiellen Energie die Winkel überhaupt nicht vorkommen, also $V=V_{1}(\varrho)+V_{2}(r)$ ist. Wir haben damit 2 voneinander unabhängige Teilchen in einem Zentralfeld, wobei die radiale Abhängigkeit des Potentials für beide Teilchen verschieden sein kann. Dieses Problem läßt sich sofort weiter lösen: Man separiert die Winkel $\vartheta_{1} \varphi_{1}$ und $\vartheta_{2} \varphi_{2}$ ab und erhält 2 unabhängige Differentialgleichungen für $\varrho$ und $r$. Als Eigenfunktion für die reduzierte Masse $\mu_{1}$ bekommen wir: $P_{i l_{1}}(\varrho) Y_{l_{1} m_{1}}\left(\vartheta_{1} \varphi_{1}\right)$ und für $\mu_{2}: R_{k l_{2}}(r) Y_{l_{2} m_{2}}\left(\vartheta_{2} \varphi_{2}\right)$. Dabei sind $Y_{l_{v} m_{v}}\left(\vartheta_{v} \varphi_{v}\right)$ die Eigenfunktionen des raumfesten Bahndrehimpulsoperators $L_{v}^{29} \cdot l_{v}$ ist die Quantenzahl des Bahndrehimpulses und $m_{v}$ die Projektion auf die raumfeste $Z$-Achse. $P_{i l_{1}}(\varrho)$ und $R_{k l_{2}}(r)$ sind die zu den speziellen Potentialen $V_{1}(\varrho)$ und $V_{2}(r)$ gehörenden Radialfunktionen. Die uns interessierenden Lösungen müssen einen scharfen Gesamtdrehimpuls mit der Quantenzahl $l$ und der Projektion $m$ auf die $Z$-Achse und Parität besitzen. Wir bilden deshalb die entsprechende Linearkombination und erhalten als Lösung für das Gesamtsystem:

$$
\begin{aligned}
& \Psi_{m}^{l l_{1} l_{2} \pm}\left(\varrho \vartheta_{1} \varphi_{1}, r \vartheta_{2} \varphi_{2}\right)=P_{i l_{1}}(\varrho) R_{k l_{2}}(r) \\
& \quad \cdot \sum_{m_{1}+m_{2}=m} C\left(l_{1} l_{2} l, m_{1} m_{2} m\right) Y_{l_{1} m_{1}}\left(\vartheta_{1} \varphi_{1}\right) Y_{l_{2} m_{2}}\left(\vartheta_{2} \varphi_{2}\right) .
\end{aligned}
$$

$C\left(l_{1} l_{2} l, m_{1} m_{2} m\right)$ sind die Clebsch-Gordan-Koeffizienten. Die Parität dieser Lösung ist $(-1)^{l_{1}+l_{2}}$, denn die Funktionen $Y_{l_{v} m_{v}}\left(\vartheta_{v} \varphi_{v}\right)$ haben in diesem Fall die Parität $(-1)^{l_{v}}$.

Nach (10) läßt sich in den Koordinaten $\varrho r u$, $\alpha \beta \gamma$ jede Lösung wie folgt schreiben:

$$
\Psi_{m}^{l_{ \pm}}(\varrho r u, \alpha \beta \gamma)=\sum_{n} \Phi_{m n}^{l_{ \pm}}(\varrho r u) \mathcal{L}_{m n}^{l_{ \pm}}(\alpha \beta \gamma) \text {. }
$$

Ein Vergleich mit der obigen Lösung im raumfesten System zeigt, daß für das spezielle von $u$ unabhängige Potential $V=V_{1}(\varrho)+V_{2}(r)$ gelten muß:

$$
\Phi_{m n}^{l \pm}(\varrho r u)=P_{i l_{1}}(r) R_{k l_{2}}(r) \chi_{m n}^{l l_{1} l_{2 \pm}}(u) .
$$


Nun hängen die Winkel $\vartheta_{1}, \varphi_{1} ; \vartheta_{2}, \varphi_{2}$ eindeutig mit den Winkeln $\alpha, \beta, \gamma, u$ zusammen, und die Funktionen $Y_{l_{1} m_{1}}\left(\vartheta_{1} \varphi_{1}\right), Y_{l_{2} m_{2}}\left(\vartheta_{2} \varphi_{2}\right)$ und $\mathcal{L}_{\text {man }}^{l \pm}(\alpha, \beta, \gamma)$ sind bekannt. Wir können also die unbekannten Funktionen $\chi_{m n}^{l_{1} l_{2} \pm}(u)$ bestimmen, indem wir die raumfeste Lösung umrechnen. Die Koordinaten $\varrho$ und $r$ werden von der Umrechnung nicht betroffen. An dieser Stelle sieht man, daß der Winkel $u$ für die Lösungstheorie der inneren Koordinaten eine besondere Rolle spielt. Wir beschäftigen uns deshalb zuerst mit der $u$-Abhängigkeit. Damit die Rechnungen übersichtlicher werden, lassen wir die $\varrho$ - und $r$ Abhängigkeit vorläufig außer Acht. Wir können sie später ohne zusätzliche Schwierigkeiten wieder mitnehmen.

\section{Umrechnung der raumfesten Lösung}

Wegen des Transformationsverhaltens der Kugelfunktionen bei Drehungen gilt:

$$
Y_{l_{2} m_{2}}\left(\vartheta_{2} \varphi_{2}\right)=\sum_{n=-l_{2}}^{l_{2}} D_{m_{2} n}^{l_{2}^{*} n}(\alpha \beta \gamma) Y_{l_{2} n}(u, 0) .
$$

Die Kugelfunktionen hängen mit den Darstellungskoeffizienten zusammen:

$$
Y_{l_{1} m_{1}}\left(\vartheta_{1} \varphi_{1}\right)=\sqrt{\frac{2 l_{1}+1}{4 \pi}} D_{m_{1} 0}^{l_{1} *}\left(\varphi_{1} \vartheta_{1} 0\right) \text {. }
$$

Da $\varphi_{1}=\alpha, \vartheta_{1}=\beta$ ist und $D_{m_{1} 0}^{l_{1} *}$ vom dritten Winkel nicht abhängt, können wir schreiben:

$$
Y_{l_{1} m_{1}}\left(\vartheta_{1} \varphi_{1}\right)=\sqrt{\frac{2 l_{1}+1}{4 \pi}} D_{m_{1} 0}^{l_{1} *}(\alpha \beta \gamma) .
$$

Wir setzen das in unsere raumfeste Lösung ein:

$$
\begin{aligned}
& \Psi_{m}^{l_{1} l_{2} \pm}\left(\vartheta_{1} \varphi_{1}, \vartheta_{2} \varphi_{2}\right)=\underset{m_{1}+m_{2}=m}{C} C\left(l_{1} l_{2} l, m_{1} m_{2} m\right) Y_{l_{1} m_{1}}\left(\vartheta_{1} \varphi_{1}\right) Y_{l_{2} m_{2}}\left(\vartheta_{2} \varphi_{2}\right) \\
& =\sum_{m_{1}+m_{2}=m} C\left(l_{1} l_{2} l, m_{1} m_{2} m\right) \sqrt{\frac{2 l_{1}+1}{4 \pi}} D_{m_{1} 0}^{l_{1} *}(\alpha \beta \gamma) \cdot \sum_{n=-l_{2}}^{l_{2}} D_{m_{2} n}^{l_{2 *}^{*}}(\alpha \beta \gamma) Y_{l_{2} n}(u, 0)=\Psi_{m}^{l_{ \pm}}(u, \alpha \beta \gamma) .
\end{aligned}
$$

Diesen Ausdruck formen wir mit der folgenden Beziehung ${ }^{9}$ weiter um:

$$
\sum_{m_{1}+m_{2}=m} C\left(l_{1} l_{2} l, m_{1} m_{2} m\right) D_{m_{1} n_{1}}^{l_{1 *}^{*}}(\alpha \beta \gamma) D_{m_{2} n_{2}}^{l_{2}^{*}}(\alpha \beta \gamma)=C\left(l_{1} l_{2} l, n_{1} n_{2} n_{1}+n_{2}\right) D_{m n_{1}+n_{2}}^{l_{2}^{*}}(\alpha \beta \gamma)
$$

und erhalten:

$$
\Psi_{m}^{\left(l_{1} l_{2} \pm\right.}(u, \alpha \beta \gamma)=\sum_{n=-l_{2}}^{l_{2}} \sqrt{\frac{2 l_{1}+1}{4 \pi}} C\left(l_{1} l_{2} l, 0 n n\right) Y_{l_{2} n}(u, 0) D_{m n}^{l^{*}}(\alpha \beta \gamma) .
$$

Um mit (10) vergleichen zu können, fassen wir die Glieder mit $n$ und $-n$ zusammen. Wir verwenden dazu:

Damit ergibt sich:

$$
\begin{aligned}
& C\left(l_{1} l_{2} l, 0-n-n\right)=(-1)^{l_{1}+l_{2}-l} C\left(l_{1} l_{2} l, 0 n n\right), \\
& Y_{l_{2}-n}(u, 0)=(-1)^{n} Y_{l_{2} n}^{*}(u, 0)=(-1)^{n} Y_{l_{2} n}(u, 0) .
\end{aligned}
$$

$$
\begin{aligned}
& \Psi_{m}^{l l_{1} l_{3} \pm}(u, \alpha \beta \gamma)=\sqrt{\frac{2 l_{1}+1}{4 \pi}} C\left(l_{1} l_{2} l, 000\right) Y_{l_{2} 0}(u, 0) D_{m 0}^{l^{*}}(\alpha \beta \gamma) \\
& +\sum_{n=1}^{l_{2}} \sqrt{\frac{2 l_{1}+1}{4 \pi}} C\left(l_{1} l_{2} l, 0 n n\right) Y_{l_{2} n}(u, 0)\left\{D_{m n}^{l^{*}}(\alpha \beta \gamma)+(-1)^{l_{1}+l_{2}}(-1)^{l+n} D_{m-n}^{l^{*}}(\alpha \beta \gamma)\right\} .
\end{aligned}
$$

Wenn wir noch berücksichtigen, daß die Parität dieser Lösung $(-1)^{l_{1}+l_{2}}$ ist, dann sehen wir, daß die Klammern $\left\{D_{m n}^{l^{*}}+(-1)^{l_{1}+l_{2}}(-1)^{l+n} D_{m-n}^{l^{*}}\right\}$ bis auf den Faktor $1 / \sqrt{2}$ bzw. $1 / 2$ die Funktionen $\mathcal{L}_{m n}^{l \pm}$ sind. Der Fall $n=0$ paßt sich dadurch an, daß für $l_{1}+l_{2}+l$ ungerade $C\left(l_{1} l_{2} l, 000\right)=0$ ist. Damit erhalten wir für die gesuchten Funktionen:

$$
\begin{aligned}
& \chi_{m n}^{l l_{1} l_{2} \pm}(u)=\sqrt{\frac{2 l_{1}+1}{2 \pi}} C\left(l_{1} l_{2} l, 0 n n\right) Y_{l_{2} n}(u, 0), n \neq 0 \\
& \chi_{m 0}^{l l_{1} l_{2} \pm}(u)=\sqrt{\frac{2 l_{1}+1}{4 \pi}} C\left(l_{1} l_{2} l, 000\right) Y_{l_{2} 0}(u, 0) .(20)
\end{aligned}
$$

Die Lösungsfunktionen mit $l_{1}+l_{2}$ gerade gehören zu $\chi_{m n}^{l l_{1} l_{2}+}(u)$, die mit $l_{1}+l_{2}$ ungerade zu $\chi_{m n}^{l_{1} l_{2}-}(u)$.

\section{Diskussion der umgerechneten Lösung}

Als erstes stellen wir fest, daß unsere umgerechneten Lösungsfunktionen $P_{i l_{1}}(\varrho) R_{k l_{2}}(r) \chi_{m n}^{l_{1} l_{2} \pm}(u)$ von der Quantenzahl $m$ nicht abhängen. Das gilt nicht nur für dieses spezielle Potential, sondern ganz allgemein, denn in den gekoppelten Differentialgleichungen für die $\Phi_{m n}^{l \pm}(\varrho r u)$ kommt $m$ nicht vor. Wir können uns das anschaulich so klarmachen: Bei einer potentiellen Energie, die von den äußeren Koordinaten $\alpha, \beta, \gamma$ unabhängig ist, können die Anteile der Wellenfunktion, die die Lage der Massen relativ zum körpergebundenen Koordinatensystem beschreiben, 
nicht von der Richtung des Gesamtdrehimpulses im Raum abhängen.

Für die weitere Diskussion formen wir die Lösung mit der folgenden Beziehung um:

$C\left(l_{1} l_{2} l, 0 n n\right)$

$$
\begin{aligned}
& =(-1)^{l_{2}+n} \sqrt{\frac{2 l+1}{2 l_{1}+1}} C\left(l l_{2} l_{1},-n n 0\right) \\
& =(-1)^{l-l_{1}+n} \sqrt{\frac{2 l+1}{2 l_{1}+1}} C\left(l l_{2} l_{1}, n-n 0\right) .
\end{aligned}
$$

Damit wird:

$$
\begin{gathered}
\Psi_{m}^{l l_{1} l_{3} \pm}(u, \alpha \beta \gamma)=(-1)^{l-l_{1}} \sum_{n=-l_{2}}^{l_{2}} C\left(l l_{2} l_{1}, n-n 0\right) \\
\cdot \sqrt{\frac{2 l+1}{8 \pi^{2}}} D_{m n}^{l_{m}^{*}}(\alpha \beta \gamma) \sqrt{2 \pi} Y_{l_{2}-n}(u, 0)
\end{gathered}
$$

Wir betrachten jetzt die einzelnen Faktoren:

$V\left(\overline{2 l+1) / 8 \pi^{2}} D_{m n}^{l^{*}}(\alpha \beta \gamma)\right.$ kennen wir bereits (5), es sind die auf 1 normierten Eigenfunktionen zum Gesamtdrehimpulsoperator $L^{2}$.

Um den Faktor $\sqrt{2 \pi} Y_{l_{2}-n}(u, 0)$ zu deuten, erinnern wir uns, woraus diese Funktionen entstanden sind. Wir haben sie erhalten, indem wir die Eigenfunktionen des Bahndrehimpulsoperators $L_{2}^{2}$ vom $X Y Z$-System auf das $\xi \eta \zeta$-System transformiert haben. Die $Y_{l_{2}-n}(u, 0)$ sind also die Eigenfunktionen von $L_{2}^{2}$ im körpergebundenen Koordinatensystem, und $-n$ ist die Projektionsquantenzahl von $\boldsymbol{L}_{2}$ auf die $\zeta$-Achse. Die Winkel $u$ und 0 sind die Polarwinkel von $\mu_{2}$ im $\xi \eta \zeta$-System. Der Azimutwinkel ist Null, weil die $\xi$-Achse durch $\mu_{2}$ gerade so definiert wird, daß $\mu_{2}$ in der $\xi-\zeta$-Ebene liegt (s. Abschnitt A III). Wir transformieren also von einem raumfesten in ein anderes verdrehtes Koordinatensystem, das sich mit $\mu_{2}$ in spezieller Weise mitbewegt. Diese Besonderheit ist auch der Grund dafür, daß nicht die Funktionen $Y_{l_{2}-n}(u, 0)$, sondern $\sqrt{2 \pi} Y_{l_{2}-n}(u, 0)$ auf 1 normiert sind, denn das Volumenelement für das Normierungsintegral lautet in unserem Fall

$$
\mathrm{d} \tau=\sin u \mathrm{~d} u \mathrm{~d} \alpha \sin \beta \mathrm{d} \beta \mathrm{d} \gamma^{4},
$$

und es gilt

$\int_{0}^{\pi} Y_{l_{2}-n}(u, 0) Y_{l_{2^{\prime}-n}}(u, 0) \sin u \mathrm{~d} u=(1 / 2 \pi) \delta_{l_{2} l_{2}}$.

Die Orthogonalität bezüglich der Quantenzahl $n$ liefern die Funktionen $D_{m n}^{l^{*}}(\alpha \beta \gamma)$.

Vergleichen wir die in den Clebsch-Gordan-Koeffizienten auftretenden Quantenzahlen $l l_{2} l_{1}, n-n 0$ mit den Quantenzahlen der Funktionen

$$
\sqrt{(2 l+1) / 8 \pi^{2}} D_{m n}^{l^{*}}(\alpha \beta \gamma) \text { und } \sqrt{2 \pi} Y_{l_{2}-n}(u, 0)
$$

und beachten die Summation über $n$, so sehen wir, daß unsere Funktion $\Psi_{m}^{l l l_{1} l_{2} \pm}(u, \alpha \beta \gamma)$ die gleiche Form hat wie eine Clebsch-Gordan-Summe, bei der aus den Produkten der Eigenfunktionen von $L^{2}$ und $L_{2}^{2}$ eine Wellenfunktion gebildet wird, die zusätzlich Eigenfunktion von $L_{1}^{2}=\left(\mathbf{L}+\mathbf{L}_{2}\right)^{2}$ ist. Das Pluszeichen zwischen $\mathbf{L}$ und $\mathbf{L}_{2}$ werden wir gleich diskutieren. Der resultierende Drehimpuls $\boldsymbol{L}_{1}$ besitzt die Projektionsquantenzahl Null auf die $\zeta$-Achse. Wir veranschaulichen uns diese Überlegungen an den Abb. 3 a und $3 \mathrm{~b}$.

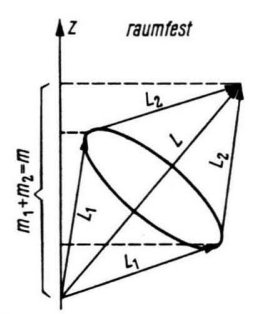

Abb. 3 a. Vektormodell für $\Psi{ }_{m}^{l l_{1} l_{2} \pm}\left(\vartheta_{1} \varphi_{1}, \vartheta_{2} \varphi_{2}\right)$.

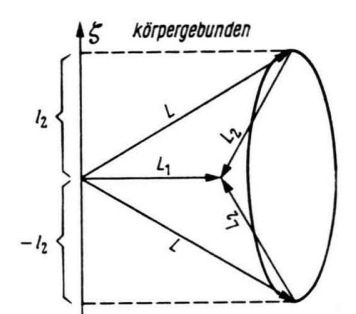

Abb. 3 b. Vektormodell für $\Psi l_{m} l_{2} \pm(u, \alpha \beta \gamma)$.
Diese Zeichnungen besitzen natürlich die üblichen Mängel solcher Vektormodelle.

Der scheinbare Widerspruch, daß es im raumfesten System $\boldsymbol{L}_{1}+\boldsymbol{L}_{\mathbf{2}}=\mathbf{L}$ heißt und im körpergebundenen System $\mathbf{L}+\mathbf{L}_{2}=\mathbf{L}_{1}$, löst sich auf, wenn wir bedenken, daß alle zu 3 a gehörenden Drehimpulsoperatoren die normalen Vertauschungsrelationen besitzen, während die Operatoren von $3 \mathrm{~b}$ zum Teil den normalen und zum Teil den anomalen Vertauschungsrelationen genügen, und zwar verhält sich, wie wir in IV sehen werden, $\boldsymbol{L}_{2}$ normal und $\boldsymbol{L}$ und $\boldsymbol{L}_{1}$ anomal. Nun bewirkt aber der Übergang eines Drehimpulses $\boldsymbol{L}_{i}$ in $-\mathbf{L}_{i}$ einen Wechsel der Vertauschungsrelationen; d. h. wir müssen $\boldsymbol{L}_{2}$ durch $-\mathbf{L}_{2}$ ersetzen, damit alle 3 Operatoren von $3 \mathrm{~b}$ die körpergebundenen anomalen Vertauschungsrelationen haben. Die $\zeta$-Komponente von $\boldsymbol{L}_{1}$ ist Null, da die Masse $\mu_{1}$ bei unserer Definition des körpergebundenen Koordinatensystems auf der $\zeta$-Achse liegt. Die $\zeta$-Komponente des Gesamtdrehimpulses wird also nur von $\boldsymbol{L}_{2}$ geliefert.

Damit können wir auch verstehen, warum die Summationsgrenze der umgerechneten Lösung $l_{2}$ ist, während bei dem allgemeinen Lösungsansatz die Summe bis $l$ läuft. Für $l<l_{2}$ sieht das zunächst wie ein Widerspruch aus; da aber die Clebsch-GordanKoeffizienten $C\left(l l_{2} l_{1}, n-n 0\right)$ für $n>\operatorname{Min}\left(l_{2}, l\right)$ Null sind, wird auch hier nur bis $l$ summiert. Für 
$l>l_{2}$ sind bei unserer speziellen Lösung alle Funktionen $\Phi_{m n}^{l \pm}(\varrho r u)$ mit $l_{2}<n \leqq l$ Null. Das liegt an dem besonderen Potential: Wir haben zwischen $\mu_{1}$ und $\mu_{2}$ keine Wechselwirkung, so daß $l_{2}$ eine feste Quantenzahl ist. Im allgemeinen Lösungsansatz dagegen sind solche Wechselwirkungen enthalten. Dadurch tritt ein Drehimpulsaustausch zwischen $\mathbf{L}_{1}$ und $\boldsymbol{L}_{2}$ auf, und $l_{2}$ kann alle Werte annehmen. Die Projektionsquantenzahl $n$ wird deshalb im allgemeinen Fall von $l$ begrenzt.

\section{Einsetzen der Lösung}

Man bekommt einen Einblick in die physikalische Bedeutung der Differentialoperatoren, wenn man ihre Wirkung auf die Funktionen $\chi_{m n}^{l l l_{1} l_{2} \pm}(u)$ untersucht. Die Koordinaten $Q$ und $r$ lassen wir dazu wie in ${ }^{4}$ mit einem Sperrpotential bei $\varrho_{0}$ bzw. $r_{0}$ starr werden. Das Sperrpotential hat die Form $V_{1}(\underline{g})$ $+V_{2}(r)$ und gehört somit zu den Potentialen, für die unsere spezielle Lösung gilt. Unser System besteht in dieser Vereinfachung aus den beiden reduzierten Massen $\mu_{1}$ und $\mu_{2}$, die in den festen Abständen $\underline{o}_{0}$ bzw. $r_{0}$ mit den Drehimpulsen $\boldsymbol{L}_{1}$ und $\boldsymbol{L}_{2}$ um den Koordinatenursprung umlaufen. In den Differentialgleichungen kommen dann die Ableitungen

nicht mehr vor.

$$
\frac{\partial}{\partial \varrho}, \frac{\partial^{2}}{\partial \varrho^{2}}, \frac{\partial}{\partial r}, \frac{\partial^{2}}{\partial r^{2}}
$$

Wir bestimmen zunächst, wie die $u$-abhängigen Operatoren auf die Funktionen $Y_{l_{2} n}(u, 0)$ wirken:

$$
\begin{aligned}
& -\hbar^{2}\left(\frac{\partial^{2}}{\partial u^{2}}+\cot u \frac{\partial}{\partial u}-\frac{n^{2}}{\sin ^{2} u}\right) Y_{l_{2} n}(u, 0)=\hbar^{2} l_{2}\left(l_{2}+1\right) Y_{l_{2} n}(u, 0), \\
& \quad\left(\frac{\partial}{\partial u}-n \cot u\right) Y_{l_{2} n}(u, 0)=\hbar \sqrt{\left(l_{2}-n\right)\left(l_{2}+n+1\right)} Y_{l_{2} n+1}(u, 0), \\
& \hbar\left(-\frac{\partial}{\partial u}-n \cot u\right) Y_{l_{2} n}(u, 0)=\hbar \sqrt{\left(l_{2}+n\right)\left(l_{2}-n+1\right)} Y_{l_{2} n-1}(u, 0) .
\end{aligned}
$$

Die $Y_{l_{2} n}(u, 0)$ sind, wie wir aus III wissen, die Eigenfunktionen des Bahndrehimpulses von $\mu_{2} \operatorname{im} \xi \eta \zeta$ System. Wir können also die 3 Operatoren auf Grund ihrer Wirkung formal als die körpergebundenen, allerdings von $n$ abhängigen Operatoren $L_{2}^{2}, L_{2+}$ und $L_{2-}$ deuten.

Bevor wir uns mit den Einzelheiten dieser Deutung näher beschäftigen, setzen wir noch die Funktionen $\chi_{m n}^{l l_{1} l_{2} \pm}(u)$ in unsere gekoppelten Differentialgleichungen ein. Mit den obigen Beziehungen wird:

$$
\begin{aligned}
& H_{i n}^{l} \chi_{m n}^{l l_{1} l_{2} \pm}(u)=\left\{\left(\frac{\hbar^{2}}{2 \mu_{1} \varrho_{0}^{2}}+\frac{\hbar^{2}}{2 \mu_{2} r_{0}^{2}}\right) l_{2}\left(l_{2}+1\right)+\frac{\hbar^{2}}{2 \mu_{1} \varrho_{0}^{2}}\left[l(l+1)-2 n^{2}\right]\right\} \chi_{m n}^{l l_{1} l_{2} \pm}(u), \\
& b_{n}^{l} \chi_{m n-1}^{l l_{1} l_{2} \pm}(u)=-\frac{\hbar^{2}}{2 \mu_{1} \varrho_{0}^{2}}[l, n]\left[l_{2}, n\right] \frac{C\left(l_{1} l_{2} l, 0 n-1 n-1\right)}{C\left(l_{1} l_{2} l, 0 n n\right)} \chi_{m n}^{l l_{1} l_{2} \pm}(u), \\
& b_{-n}^{l} \chi_{m n+1}^{l l_{1} l_{2} \pm}(u)= \\
& \frac{\hbar^{2}}{2 \mu_{1} \varrho_{0}^{2}}[l,-n]\left[l_{2},-n\right] \frac{C\left(l_{1} l_{2} l, 0 n+1 n+1\right)}{C\left(l_{1} l_{2} l, 0 n n\right)} \chi_{m n}^{l l_{1} l_{2} \pm}(u)
\end{aligned}
$$

mit der Abkürzung: $\quad[l, n]$ Def. $\sqrt{(l+n)(l-n+1})$.

Die $n$-te Zeile unseres Differentialgleichungssystems ergibt dann:

$$
\begin{aligned}
0= & b_{n}^{l} \chi_{m n-1}^{l l_{1} l_{2} \pm}(u)+\left(H_{i n}^{l}-E\right) \chi_{m n}^{l l_{1} l_{2} \pm}(u)-b_{-n}^{l} \chi_{m n+1}^{l l_{1} l_{2} \pm}(u) \\
= & \left\{\frac{\hbar^{2}}{2 \mu_{2} r_{0}^{2}} l_{2}\left(l_{2}+1\right)+\frac{\hbar^{2}}{2 \mu_{1} \varrho_{0}^{2}}\left[\left(l_{2}\left(l_{2}+1\right)+l(l+1)-2 n^{2}\right) C\left(l_{1} l_{2} l, 0 n n\right)\right.\right. \\
& \left.-[l, n]\left[l_{2}, n\right] C\left(l_{1} l_{2} l, 0 n-1 n-1\right)-[l,-n]\left[l_{2},-n\right] C\left(l_{1} l_{2} l, 0 n+1 n+1\right)\right] \\
& \left.\cdot \frac{1}{C\left(l_{1} l_{2} l, 0 n n\right)}-E\right\} \chi_{m n}^{l l_{1} l_{2} \pm}(u)=0 .
\end{aligned}
$$

Der Energieeigenwert $E$ hängt hier scheinbar noch von $n$ ab. Da aber die Clebsch-Gordan-Koeffizienten die Rekursionsbeziehung ${ }^{9}$

$$
\begin{aligned}
& {\left[-l_{1}\left(l_{1}+1\right)+l_{2}\left(l_{2}+1\right)+l(l+1)-2 n^{2}\right] C\left(l_{1} l_{2} l, 0 n n\right)} \\
& \quad-[l, n]\left[l_{2}, n\right] C\left(l_{1} l_{2} l, 0 n-1 n-1\right)-[l,-n]\left[l_{2},-n\right] C\left(l_{1} l_{2} l, 0 n+1 n+1\right)=0
\end{aligned}
$$


erfüllen, erhalten wir:

$$
E=\frac{\hbar^{2}}{2 \mu_{1} \varrho_{0}^{2}} l_{1}\left(l_{1}+1\right)+\frac{\hbar^{2}}{2 \mu_{2} r_{0}^{2}} l_{2}\left(l_{2}+1\right) .
$$

Dieser Ausdruck ist für jede Zeile der gleiche. Das richtige Verhalten der Lösung an den Rändern des Gleichungssystems wird durch die Symmetrien der Clebsch-Gordan-Koeffizienten geregelt. Unser Differentialgleichungssystem ist damit gelöst.

Das Ergebnis für den Energieeigenwert ist unmittelbar verständlich. Wir bemerken noch, daß $E$ die Form $E_{1}(\varrho)+E_{2}(r)$ hat. Wenn wir nun die $\varrho$ und $r$-Abhängigkeit mitnehmen, erhalten wir genau wie im raumfesten Fall 2 unabhängige Differentialgleichungen für $\varrho$ und $r$.

Die in (20) gefundene Lösung für die $u$-Abhängigkeit gilt, wie aus der Herleitung hervorgeht, nicht nur für potentielle Energien der Form $V_{1}(\varrho)+V_{2}(r)$, sondern für jede von $u$ unabhängige potentielle Energie $V(\varrho, r)$. Die Differentialgleichungen für $\varrho$ und $r$ sind dann natürlich über $V(\varrho, r)$ gekoppelt. Ein Spezialfall ist das von Zickendraht ${ }^{5,6}$ verwendete Potential $V(y)$, da $y$ mit unseren Koordinaten verknüpft ist durch

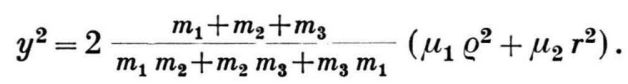

Ein solches Potential $V(y)$ hat allerdings keine unmittelbare physikalische Bedeutung.

Wir wenden uns jetzt wieder der Deutung unserer Operatoren zu. Dazu betrachten wir einen Teil des Hamilton-Operators (3), an dem wir bereits alles Wesentliche diskutieren können.

$$
\begin{gathered}
-\frac{\hbar}{i} e^{-i \gamma}\left[\cot \beta \frac{\partial}{\partial \gamma}+i \frac{\partial}{\partial \beta}-\frac{1}{\sin \beta} \frac{\partial}{\partial \alpha}\right] \\
\cdot\left[-\cot u \frac{\hbar}{i} \frac{\partial}{\partial \gamma}-\hbar \frac{\partial}{\partial u}\right] .
\end{gathered}
$$

In Abschnitt B haben wir für $(\hbar / i) e^{-i \gamma}$ mal der ersten Klammer den körpergebundenen Schrauboperator $L_{+}$eingeführt. Nachdem dann der HamiltonOperator auf unseren Lösungsansatz gewirkt hatte, konnten wir die zweite Klammer formal als den von $n$ abhängigen $L_{2-}$-Operator für die Funktionen $Y_{l_{2} n}(u, 0)$ deuten. Jetzt sehen wir noch eine andere Möglichkeit: Wir verwenden die Beziehung

$$
Y_{l_{2} n}(u, 0) D_{m n}^{l^{*}}(\alpha \beta \gamma)=Y_{l_{2} n}(u, \gamma) D_{m n}^{l^{*}}(\alpha \beta 0),
$$

d. h. wir nehmen $e^{i n \gamma}$ von $D_{m n}^{l_{m}^{*}}(\alpha \beta \gamma)$ weg, schreiben es zu $Y_{l_{2} n}(u, 0)$ und erhalten $Y_{l_{2} n}(u, \gamma)$. Für diese Funktion ist dann $e^{-i \gamma}$ mal der zweiten Klammer der normale, von $n$ unabhängige Schrauboperator $L_{2}$. Die erste Klammer dagegen bekommt für $D_{m n}^{l^{*}}(\alpha \beta 0)$ erst dann die Bedeutung von $L_{+}$, wenn der gesamte Operator auf $Y_{l_{2} n}(u, \gamma)$ gewirkt hat. Da nur Produkte $Y_{l_{2} n} D_{m n}^{l^{*}}$ mit gleichem $n$ auftreten $\left(Y_{l_{2}-n}(u, 0)=(-1)^{n} Y_{l_{2} n}(u, 0)\right)$, stimmt das $n$ in den Operatoren mit dem $n$ der dazugehörigen Funktionen immer überein. An dieser Stelle erkennen wir die Doppelrolle von $\gamma$ : Einerseits gehört $\gamma$ zu den Eulerschen Winkeln, die die Lage des $\xi \eta \zeta$-Systems definieren, und andererseits ist $\gamma$ der Azimutwinkel von $\mu_{2}$. Die erste Möglichkeit haben wir ausgeführt, indem wir das körpergebundene Koordinatensystem durch die Lage von $\mu_{1}$ und $\mu_{2}$ festgelegt haben. Den zweiten Fall erhält man, wenn man das $\xi \eta \zeta$-System allein durch die Lage von $\mu_{1}$ bestimmt, und zwar so, daß die $\xi$-Achse in der $Z$ - $\zeta$-Ebene liegt. Die Eulerschen Winkel sind dann $\alpha, \beta, 0$; der Winkel $\gamma$ ist in diesem System der Azimutwinkel von $\mu_{2}$. Beide Möglichkeiten sind aus dieser Sicht gleichberechtigt. Da wir aber wegen der potentiellen Energie zwischen äußeren und inneren Koordinaten unterscheiden, ist es für unser Problem sinnvoller, $\alpha \beta \gamma$ beisammen zu lassen und erst die Funktionen $\mathcal{L}_{m n}^{l \pm}(\alpha \beta \gamma)$ abzuseparieren.

Mit diesen Überlegungen ist die zunächst formale Einführung von $L_{2}^{2}, L_{2+}$ und $L_{2}$ für die $n$-abhängigen Operatoren verstanden. Die auftretenden Schwierigkeiten liegen also an der Doppelrolle von $\gamma$; wenn wir sie beachten, können wir für den betrachteten Operator im weiteren $-L_{+} L_{2}$ schreiben.

An der Gestalt und dem Schraubsinn von $L_{2-}$ sehen wir, daß sich $\boldsymbol{L}_{2}$ wie ein normaler Drehimpulsoperator verhält; dieses Ergebnis haben wir in III bereits vorweggenommen. In diesem Zusammenhang betrachten wir noch einmal die Wirkung von $-L_{+} L_{2}$ auf die Funktionen $Y_{l_{2} n} D_{m n}^{l^{*}}$. Da sich die Komponenten von $\boldsymbol{L}$ anomal und die von $\boldsymbol{L}_{2}$ normal verhalten, schrauben $L_{+}$und $L_{2-}$ in dieselbe Richtung, und wir bekommen

$$
-\hbar^{2}[l, n]\left[l_{2}, n\right] Y_{l_{2} n-1} D_{m n-1}^{l^{*}},
$$

also wieder ein Produkt von Eigenfunktionen, bei denen die Projektionsquantenzahlen auf die $\zeta$-Achse gleich sind.

Die Reihenfolge der Operatoren $L_{+}$und $L_{2}$ - wird jetzt ebenfalls verständlich. Dazu stellen wir zunächst fest, daß $e^{-i \gamma}$ mit keiner der beiden Klammern ver- 
tauschbar ist, wohl aber die Klammern unter sich. Das heißt, je nachdem wie wir $\gamma$ deuten wollen, vertauschen wir die beiden Klammern, fassen die an erster Stelle stehende Klammer mit $e^{-i \gamma}$ zusammen und erhalten entweder $-L_{+} L_{2-}$ oder $-L_{2_{-}} L_{+}$. Der erste Operator des Produkts ist dann immer der vollständige Schrauboperator. In diesem Sinne sind die Operatoren $L_{+}$und $L_{2-}$ sogar vertauschbar.

\section{F. Zusammenfassung der Lösungstheorie}

Wir gehen noch einen Schritt weiter und führen die Bahndrehimpulsoperatoren von $\mu_{2}$ in die Schrödinger-Gleichung (4) ein, also noch bevor wir den Hamilton-Operator auf den Lösungsansatz anwenden. Dieser Schritt liefert uns eine einprägsame Gestalt des Hamilton-Operators, an der wir außerdem einige allgemeine Eigenschaften ablesen können. Wenn wir jedoch mit den Operatoren rechnen, müssen wir auf die in Abschnitt EIV diskutierte Reihenfolge achten und auf unsere bisherigen Ergebnisse zurückgreifen.

Für den Hamilton-Operator erhalten wir:

$$
\begin{aligned}
& \frac{1}{2 \mu_{1}}\left\{-\hbar^{2}\left(\frac{\partial^{2}}{\partial \varrho^{2}}+\frac{2}{\varrho} \frac{\partial}{\partial \varrho}\right)+\frac{1}{\varrho^{2}}\left(L_{2}^{2}+L^{2}-2 L_{\zeta}^{2}-L_{+} L_{2-}-L_{-} L_{2+}\right)\right\} \\
& +\frac{1}{2 \mu_{2}}\left\{-\hbar^{2}\left(\frac{\partial^{2}}{\partial r^{2}}+\frac{2}{r} \frac{\partial}{\partial r}\right)+\frac{1}{r^{2}} L_{2}^{2}\right\}+V(\varrho r u)
\end{aligned}
$$

Nun läßt sich der Operator $2 L_{\zeta}^{2}$ wegen $L_{\zeta}=L_{2 \zeta}$ durch $2 L_{\zeta} L_{2 \zeta}$ ersetzen. Damit können wir dann für die Klammer $\left(L_{2}^{2}+L^{2}-L_{+} L_{2-}-L_{-} L_{2+}-2 L_{\zeta}^{2}\right)$ formal $\left(\boldsymbol{L}-\mathbf{L}_{2}\right)^{2}$ schreiben. Wir führen noch die Abkürzung

$$
[\varrho] \underset{\text { Def. }}{\overline{=}}-\hbar^{2}\left(\frac{\partial^{2}}{\partial \varrho^{2}}+\frac{2}{\varrho} \frac{\partial}{\partial \varrho}\right)
$$

ein und stellen die körpergebundene und raumfeste Darstellung einander gegenüber:

\section{Körpergebunden:}

Koordinaten $\varrho r u, \alpha \beta \gamma$;

$$
H=\frac{1}{2 \mu_{1}}\left\{[\varrho]+\frac{1}{\varrho^{2}}\left(\mathbf{L}-\mathbf{L}_{2}\right)^{2}\right\}+\frac{1}{2 \mu_{2}}\left\{[r]+\frac{1}{r^{2}} L_{2}^{2}\right\}+V(\varrho r u) .
$$

Lösung für $V(\varrho r u)=V_{1}(\varrho)+V_{2}(\varrho)$

$$
\begin{aligned}
& \Psi_{m}^{l l_{1} l_{2} \pm}(\varrho r u, \alpha \beta \gamma)= \\
& \quad P_{i l_{1}}(\varrho) R_{k l_{2}}(r) \sum_{n=-l_{2}}^{l_{2}}(-1)^{l-l_{1}} C\left(l l_{2} l_{1}, n-n 0\right) \sqrt{\frac{2 l+1}{8 \pi^{2}}} D_{m n}^{l^{*}}(\alpha \beta \gamma) \sqrt{2 \pi} Y_{l_{2}-n}(u, 0) .
\end{aligned}
$$

Raumfest:

Koordinaten $\varrho \vartheta_{1} \varphi_{1}, r \vartheta_{2} \varphi_{2}$;

$$
H=\frac{1}{2 \mu_{1}}\left\{[\varrho]+\frac{1}{\varrho^{2}} L_{1}^{2}\right\}+\frac{1}{2 \mu_{2}}\left\{[r]+\frac{1}{r^{2}} L_{2}^{2}\right\}+V\left(\varrho \vartheta_{1} \varphi_{1}, r \vartheta_{2} \varphi_{2}\right) .
$$

Lösung für $V\left(\varrho \vartheta_{1} \varphi_{1}, r \vartheta_{2} \varphi_{2}\right)=V_{1}(\varrho)+V_{2}(r)$

$$
\Psi_{m}^{l l_{1} l_{2} \pm}\left(\varrho \vartheta_{1} \varphi_{1}, r \vartheta_{2} \varphi_{2}\right)=P_{i l_{1}}(\varrho) R_{k l_{2}}(r) \underset{m_{1}+m_{2}=m}{C} \underset{l_{1}}{C}\left(l_{1} l_{2} l, m_{1} m_{2} m\right) Y_{l_{1} m_{1}}\left(\vartheta_{1} \varphi_{1}\right) Y_{l_{2} m_{2}}\left(\vartheta_{2} \varphi_{2}\right) .
$$

Man erhält also den körpergebundenen Fall formal aus dem raumfesten, indem man $\mathbf{L}_{1}$ durch $\mathbf{L}-\mathbf{L}_{2}$ ersetzt, und die Drehimpulse $\mathbf{L}$ und $\mathbf{L}_{2}$ auf die Achsen des körpergebundenen Koordinatensystems zerlegt. Dieses Ergebnis ist vom Standpunkt der klassischen Mechanik völlig klar. In der Quantenmechanik jedoch ist bei der Zerlegung von Drehimpulsoperatoren auf mitbewegte Achsen Vorsicht geboten, wie in ${ }^{7}$ diskutiert wurde. 
An dieser Gestalt des Hamilton-Operators sehen wir auch den Unterschied zwischen $\varrho$ - und $r$-RichtungQuantisierung: Wenn wir die körpergebundene Quantisierungsachse in die $\varrho$-Richtung legen, tritt $\mathbf{L}-\mathbf{L}_{2}$ an die Stelle von $\mathbf{L}_{1}$, wählen wir die $r$-Richtung, so steht $\mathbf{L}-\mathbf{L}_{1}$ an Stelle von $\mathbf{L}_{2}$. Bei unserem speziellen Potential ist keine der beiden Möglichkeiten ausgezeichnet.

Wir betrachten nun ein allgemeines Potential $V(\varrho r u)$. Da dann zwischen $\mu_{1}$ und $\mu_{2}$ eine Wechselwirkung vorhanden ist, sind $\mathbf{L}_{1}$ und $\mathbf{L}_{2}$ nicht mehr scharf, und wir müssen über alle Drehimpulsquantenzahlen $l_{1}$ und $l_{2}$ summieren. Dasselbe gilt auch für die radialen Quantenzahlen $i$ und $k$. Die allgemeine Lösung sieht daher bei $\varrho$-Richtung-Quantisierung wie folgt aus:

$\Psi_{m}^{l}(\varrho r u, \alpha \beta \gamma)=\sum_{k l_{2}} a_{k l_{2}} R_{k l_{2}}(r) \sum_{i l_{1}} b_{k l_{2} i l_{1}} P_{i l_{1}}(\varrho) \sum_{n=-l_{2}}^{l_{2}} C\left(l l_{2} l_{1}, n-n 0\right) \sqrt{2 \pi} Y_{l_{2}-n}(u, 0) \sqrt{\frac{2 l+1}{8 \pi^{2}}} D_{m n}^{l^{*}}(\alpha \beta \gamma)$.

Weil der von $u$ abhängige Teil der Lösung von der Summation über $l_{1}$ nicht betroffen wird, fassen wir $\Psi_{m}^{l}$ dementsprechend zusammen. Außerdem können wir für die Summationsgrenzen von $n$ auch $l$ an Stelle von $l_{2}$ schreiben, wie wir uns in Abschnitt E III überlegt haben.

$\Psi_{n b}^{l}(\varrho r u, \alpha \beta \gamma)=\sum_{k l_{2}} a_{k l_{2}} R_{k l_{2}}(r) \sum_{n=-l}^{l}\left\{\sum_{i l_{1}} b_{k l_{2} i l_{1}} P_{i l_{1}}(\varrho) C\left(l l_{2} l_{1}, n-n 0\right)\right\} \sqrt{2 \pi} Y_{l_{2}-n}(u, 0) \sqrt{\frac{2 l+1}{8 \pi^{2}}} D_{m n}^{l_{m}^{*}}(\alpha \beta \gamma)$.

Die in der Klammer stehende Doppelsumme über $i$ und $l_{1}$ können wir nun auch durch eine einfache Summe über $i^{\prime}$ ersetzen, indem wir die $\varrho$-Abhängigkeit nach einem neuen vollständigen Funktionensystem $P_{i^{\prime} k l_{2} n}^{\prime}(\varrho)$ entwickeln. Zusätzlich ordnen wir die Summen über $l_{2}$ und $n$ um und erhalten:

$$
\Psi_{m}^{l}(\varrho r u, \alpha \beta \gamma)=\sum_{n=-l}^{l}\left\{\sum_{i^{\prime} k ; l_{2} \geq|n|} a_{i^{\prime} k l_{2} n}^{\prime} R_{k l_{2}}(r) P_{i^{\prime} k l_{2} n}^{\prime}(\varrho) \sqrt{2 \pi} Y_{l_{2}-n}(u, 0)\right\} \sqrt{\frac{2 l+1}{8 \pi^{2}}} D_{m n}^{l^{*}}(\alpha \beta \gamma) .
$$

Wir haben also die Entwicklung nach Eigenzuständen von $i, k, l_{1}, l_{2}$ durch die Entwicklung nach $i^{\prime}, k, l_{2}, n$ ersetzt, und es werden hier für jedes $l_{2}$ die dazugehörenden $l_{1}$-Werte exakt mitgenommen.

Im Falle der $r$-Richtung-Quantisierung können wir analog vorgehen und erhalten dann eine Entwicklung nach Eigenzuständen von $i, k^{\prime}, l_{1}, n^{\prime}$, wobei $n^{\prime}$ die Projektion von $\mathbf{L}_{1}$ bzw. $\mathbf{L}$ auf die $r$-Richtung ist.

Mit diesen Überlegungen können wir jetzt in vielen Fällen entscheiden, welche Richtung für die $\zeta$ Achse günstiger ist. Wir betrachten dazu die Energieeigenwerte der Funktionen, nach denen wir entwickeln, in Abhängigkeit von $l_{2}$ und $n$ bzw. $l_{1}$ und $n^{\prime}$. Wenn beispielsweise die Energiestufen von $l_{1}$ dichter liegen als die von $l_{2}$, ist es vorteilhafter, nach $l_{2} \mathrm{zu}$ entwickeln, also die $\varrho$-Richtung zu wählen, denn dann ist $l_{2}$ in besserer Näherung als $l_{1}$ noch eine Quantenzahl, und man kommt bei $l_{2}$ mit weniger Entwicklungskoeffizienten aus. Dieses Ergebnis werden wir in Abschnitt $G$ verwenden.

Im allgemeinen nehmen wir die Summation über $n$ exakt mit, da sie endlich ist. Nun gibt es aber auch wichtige Fälle in der Molekülphysik und bei den Kernmodellen, bei denen $n$ in guter Näherung eine Quantenzahl ist, da die Zustände mit verschiedenem $n$ energetisch weit auseinander liegen. Da man dann mit einem oder nur wenigen $n$ auskommt, ist es hier besonders vorteilhaft, die $\varrho$-Richtung als $\zeta$-Achse zu verwenden.

Die Entscheidung zwischen $\varrho$ - und $r$-Richtung läßt sich auch anschaulich verstehen. Etwas vereinfachend kann man sagen, daß in vielen Fällen dichtliegende Energiestufen klassisch großen Trägheitsmomenten und damit langsamer Rotation entsprechen. Unsere Betrachtungen besagen dann in diesem Bild, daß diejenige Richtung für die $\zeta$-Achse günstiger ist, die langsamer rotiert.

Die bisherigen Ergebnisse gelten für 3 beliebige Massen. Für die Praxis ist jedoch der Fall, daß Teilchen 1 und 2 gleich sind, besonders wichtig. In $\mathrm{Ab}$ schnitt C III haben wir die Vertauschung dieser beiden Teilchen allgemein behandelt. Wir wenden jetzt die Resultate auf unseren Lösungsansatz an. Es ist

$$
\begin{aligned}
E_{12} Y_{l_{2} n}(u, 0) & =Y_{l_{2} n}(\pi-u, 0) \\
& =(-1)^{l_{2}+n} Y_{l_{2} n}(u, 0) .
\end{aligned}
$$

Da für den symmetrischen Zustand

$$
E_{12} \Phi_{m n}^{l s \pm}= \pm(-1)^{n} \Phi_{m n}^{l s \pm}
$$

und für den antisymmetrischen

$$
E_{12} \Phi_{m n}^{l a \pm}=\mp(-1)^{n} \Phi_{m n}^{l a \pm}
$$


sein muß, folgt daraus, daß in einer Lösung mit gegebener Symmetrie bezüglich Teilchenvertauschung nur gerade oder nur ungerade Bahndrehimpulsquantenzahlen $l_{2}$ vorkommen dürfen, und zwar enthält bei positiver Parität die symmetrische Lösung nur gerade $l_{2}$, die antisymmetrische Lösung nur ungerade $l_{2}$; bei negativer Parität ist es genau umgekehrt. Die Teilchensymmetrie reduziert also die Entwicklungsfunktionen für einen Zustand mit bestimmter Symmetrie auf die Hälfte. Im nächsten Abschnitt werden wir das an einem Beispiel ausführlich diskutieren.

\section{G. Lösungspraxis}

In diesem Abschnitt verwenden wir unsere Ergebnisse, um die gekoppelten Differentialgleichungssysteme für konkrete Potentiale numerisch zu lösen. Dazu entwickeln wir die gesuchten Wellenfunktionen nach geeignet gewählten Funktionen aus Abschnitt $\mathrm{F}$ und bestimmen die Entwicklungskoeffizienten sowie den Energieeigenwert, indem wir die auftretenden Matrizen diagonalisieren. Die erforderlichen Rechnungen wurden auf einer Siemens DVA 2002 durchgeführt.

Damit man an einem übersichtlichen Beispiel sieht, wie dieses Verfahren im Falle unserer gekoppelten
Differentialgleichungen aussieht, beginnen wir hier genauso wie in der Lösungstheorie mit einem System, bei dem $\varrho$ und $r$ starr sind.

$$
\text { I. } \varrho \text { und } r \text { starr } ; V=V(u) \text {. }
$$

Beispiel $V(u)=\delta\left(\cos ^{2} u-1 / 3\right)$

Wir betrachten im folgenden nur Systeme, bei denen die beiden Teilchen 1 und 2 gleich sind. Die potentielle Energie muß dann die Eigenschaft $V(u)=V(\pi-u)$ haben. Für $u$-unabhängiges Potential konnten wir das Problem exakt lösen. Es waren dabei $\mathbf{L}_{1}, \mathbf{L}_{2}$ und $\mathbf{L}$ scharf. Jetzt ist nur noch der Gesamtdrehimpuls $\boldsymbol{L}$ scharf. Da keine Funktionen von $\varrho$ und $r$ vorhanden sind, erhalten wir die vollständige Entwicklung unserer Lösung, wenn wir mit unbestimmten Koeffizienten über einen der beiden Drehimpulse summieren. Die Wahl der Quantisierungsachse richtet sich hier nach dem Verhältnis der beiden Trägheitsmomente $\mu_{1} \varrho_{0}^{2}$ und $\mu_{2} r_{0}^{2}$. Wir wählen $\mu_{1} \varrho_{0}^{2}>\mu_{2} r_{0}^{2}$ und entwickeln daher nach $l_{2}$. Die Eigenzustände werden nach Gesamtdrehimpuls, Parität und Symmetrie bezüglich Teilchenvertauschung klassifiziert.

Da die Differentialgleichungen nur über die Koordinate $u$ verkoppelt sind, zeigt unser einfaches Beispiel bereits alles Wesentliche; wir schreiben deshalb die auftretenden Funktionen ausführlich hin:

$l$ gerade $\left(l_{2} \geqq n\right)$ :

$$
\begin{aligned}
& \Psi_{m}^{l+}=\sum_{n=0}^{l} \Phi_{m n}^{l+} \mathcal{L}_{m n}^{l+} \backslash \begin{array}{l}
\Psi_{m}^{l s+}=\sum_{n=0}^{l}\left(\sum_{l_{2}=0,2,4, \ldots} c_{l_{2} n}^{l s+} Y_{l_{2} n}(u, 0)\right) \mathcal{L}_{m n}^{l+}, \\
\Psi_{m}^{l a+}=\sum_{n=0}^{l}\left(\sum_{l_{2}=1,3,5, \ldots} c_{l_{2} n}^{l a+} Y_{l_{2} n}(u, 0)\right) \mathcal{L}_{m n}^{l+},
\end{array} \\
& \Psi_{m}^{l-}=\sum_{n=1}^{l} \Phi_{m n}^{l-} \mathcal{L}_{m n}^{l-} \backslash \Psi_{m}^{l s-}=\sum_{n=1}^{l}\left(\sum_{l_{2}=1,3,5, \ldots} c_{l_{2} n}^{l s-} Y_{l_{2} n}(u, 0)\right) \mathcal{L}_{m n}^{l-}, \\
& \Psi_{m}^{l a-}=\sum_{n=1}^{l}\left(\sum_{l_{2}=2,4,6, \ldots} c_{l_{2} n}^{l a-} Y_{l_{2} n}(u, 0)\right) \mathcal{L}_{m n}^{l-} .
\end{aligned}
$$

Für $l$ ungerade erhält man die gleichen Fälle, und zwar entspricht

$$
\begin{aligned}
& (l \text { ungerade, } s,+) \triangleq(l \text { gerade }, a,-), \\
& (l \text { ungerade }, a,+) \triangleq(l \text { gerade, } s,-), \\
& (l \text { ungerade } s,-) \triangleq(l \text { gerade, } a,+), \\
& (l \text { ungerade }, a,-) \triangleq(l \text { gerade, } s,+) .
\end{aligned}
$$

Die zu diagonalisierenden Matrizen entstehen, indem wir die Funktionen

$$
\Phi_{m n}^{l_{m}^{ \pm}}=\sum_{l_{s}} c_{l_{2} n}^{l_{a}^{ \pm} \pm} Y_{l_{2} n}(u, 0)
$$

in die gekoppelten Differentialgleichungen (13) bzw. (14) einsetzen, von links mit $Y_{l_{2}^{\prime} n^{\prime}}(u, 0)$ multiplizieren und dann mit dem Faktor $\sin u \mathrm{~d} u$ integrieren, und zwar muß, wegen der Orthogonalität der Funktionen $\mathcal{L}_{m n}^{l \pm}(\alpha \beta \gamma)$ bezüglich $n$ die $n$-te Zeile nur mit $Y_{l_{2}^{\prime} n}(u, 0), l_{2}^{\prime} \geqq n$, multipliziert werden. Die Wirkung der Operatoren $H_{i n}^{l}, b_{n}^{l}, b_{-n}^{l}$ auf $Y_{l_{2} n}(u, 0)$ kennen wir von Abschnitt $E$ IV. Wir führen noch die folgenden Abkürzungen ein: 


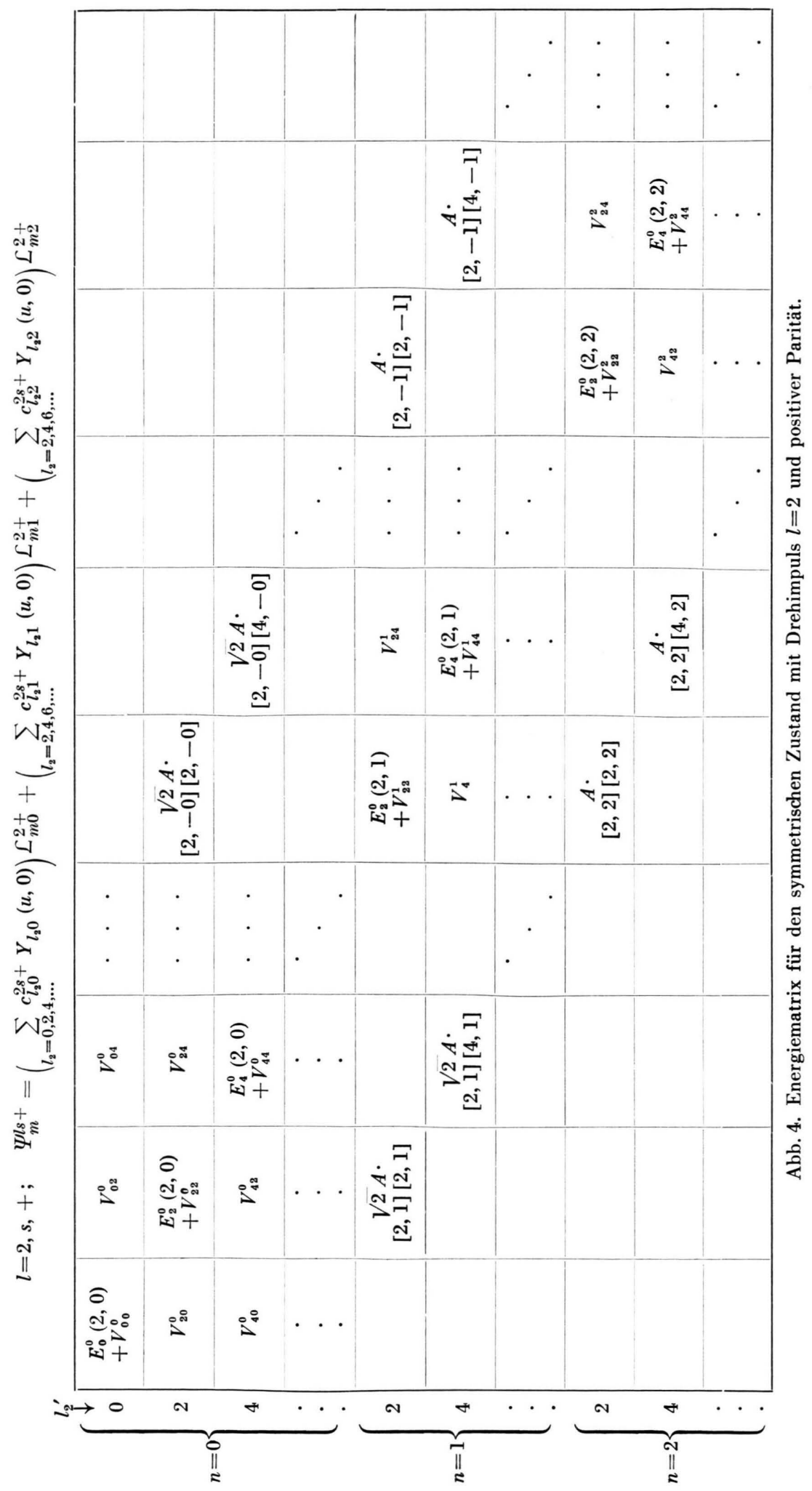




$$
\begin{gathered}
E_{l_{2}}^{0}(l, n) \underset{\text { Def. }}{=} \frac{\hbar^{2}}{2 \mu_{1} \varrho_{0}^{2}}\left[l_{2}\left(l_{2}+1\right)+l(l+1)-2 n^{2}\right] \\
\quad+\frac{\hbar^{2}}{2 \mu_{2} r_{0}^{2}} l_{2}\left(l_{2}+1\right), \\
V_{l_{2} l_{2}}^{n} \underset{\text { Def. }}{=} 2 \pi \int_{0}^{\pi} Y_{l_{2}^{\prime} n}(u, 0) V(u) Y_{l_{2} n}(u, 0) \sin u \mathrm{~d} u, \\
A \underset{\text { Def. }}{=} \frac{\hbar^{2}}{2 \mu_{1} \varrho_{0}^{2}}, \quad[l, n]=\sqrt{(l+n)(l-n+1)}
\end{gathered}
$$

und geben in Abb. 4 als Beispiel die Matrix für $l=2, s,+$ an (Abb. 4 siehe vorhergehende S. 593).

Die Matrizen besitzen für alle Kombinationen von $l\left(\begin{array}{l}\boldsymbol{s} \\ a\end{array}\right)\left(\begin{array}{c} \pm \\ -\end{array}\right)$ dieselbe Struktur: Um die Hauptdiagonale stehen für jedes $n$ quadratische Untermatrizen, deren Elemente vom Potential, aber nicht von $l$ abhängen. Die Untermatrix $n$ ist über "Nebendiagonalen" mit den Untermatrizen $n-1$ und $n+1$ verkoppelt. Die Quantenzahl $l$ kommt nur noch in den Nebendiagonalen und in der Hauptdiagonale vor, und zwar in ganz einfacher Weise. Darin liegt der große Vorteil dieser Entwicklung gegenüber der Entwicklung nach Eigenfunktionen von $l_{1}$ und $l_{2}$, die für unser System die Form

$$
\sum_{l_{1} l_{2}} \boldsymbol{a}_{l_{1} l_{2}}\left\{\sum_{n} C\left(l l_{2} l_{1}, n-n 0\right) Y_{l_{2}-n}(u, 0) D_{m n}^{l^{*}}(\alpha \beta \gamma)\right\}
$$

hat und die als Matrixelemente

$V_{l_{1}^{\prime} l_{2}^{\prime} l_{1} l_{2}}=\sum_{n} C\left(l l_{2}^{\prime} l_{1}^{\prime}, n-n 0\right) C\left(l l_{2} l_{1}, n-n 0\right) V_{l^{\prime} 2 l_{2}}^{n}$ liefert, also Matrixelemente, die nicht nur komplizierter, sondern auch für jedes $l$ verschieden sind. Für Diagonalisierungsverfahren ist daher immer unsere Methode besser; die zweite Möglichkeit ist dagegen für eine erste Näherung geeignet, weil man hier für das ungestörte Problem eine Diagonalmatrix erhält.

An unseren Matrizen erkennen wir wieder unmittelbar die Bedeutung der $\varrho$ - bzw. $r$-RichtungQuantisierung. Im einen Fall enthalten die Nebendiagonalen den Faktor $\hbar^{2} /\left(2 \mu_{1} \varrho_{0}^{2}\right)$, im anderen $\hbar^{2} /\left(2 \mu_{2} r_{0}^{2}\right)$. Es hängt somit direkt von der Größe der Trägheitsmomente $a b$, wie stark die Zustände mit verschiedenem $n$ bzw. $n^{\prime}$ untereinander über die Nebendiagonalen verkoppelt sind.

Als spezielles Beispiel wählen wir

$$
V(u)=\delta\left(\cos ^{2} u-\frac{1}{3}\right)=\delta \frac{4}{3} \sqrt{\frac{\pi}{5}} Y_{20}(u, 0)
$$

und

$$
\frac{\mu_{1} \varrho_{0}^{2}}{\mu_{2} r_{0}^{2}}=5
$$

Die Niveaus sind in Abb. 5 als Funktion von $\delta$ aufgetragen. Für $\delta=0$ ist

$$
E=\frac{\hbar^{2} l_{1}\left(l_{1}+1\right)}{2 \mu_{1} \varrho_{0}^{2}}+\frac{\hbar^{2} l_{2}\left(l_{2}+1\right)}{2 \mu_{2} r_{0}^{2}}
$$

und wir haben Entartung, da zu gegebenem $l_{1}$ und $l_{2}$ alle Gesamtdrehimpulse mit $\left|l_{1}-l_{2}\right| \leqq l \leqq l_{1}+l_{2}$ gehören. Diese Entartung wird für $\delta \neq 0$ aufgeho-

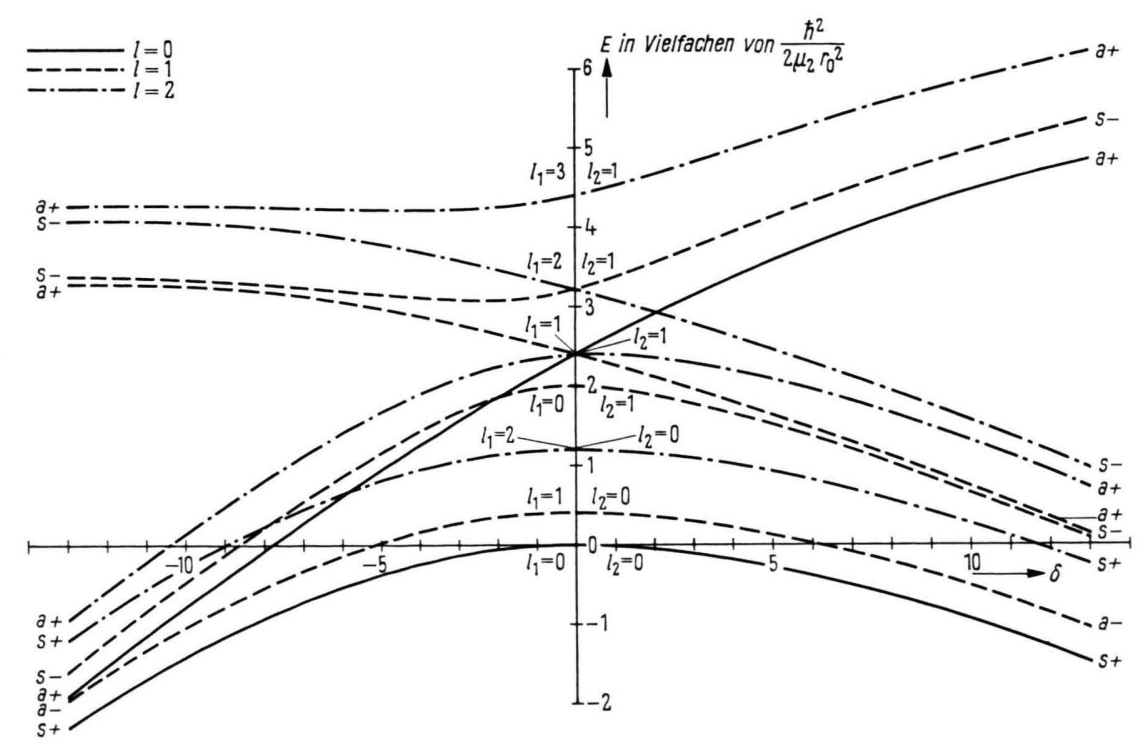

Abb. 5. Energieniveaus für das Potential $V(u)=\delta\left(\cos ^{2} u-1 / 3\right)$ als Funktion von $\delta$. 
ben. Für große $\delta$ oder kleine $\hbar^{2} /\left(2 \mu_{1} \varrho_{0}^{2}\right)$, das heißt, wenn die Matrixelemente $V_{l^{\prime}{ }_{2} l_{2}}^{n}$ größer werden als die in den Nebendiagonalen, erhalten wir näherungsweise die Niveaus, die durch Diagonalisierung der $n$-Untermatrizen für sich allein entstehen würden. Diese Niveaus unterscheiden sich für gleiches $n$ aber verschiedenes $l$ wegen der Form von $E_{l_{2}}^{0}(l, n)$ nur durch $\left[\hbar^{2} /\left(2 \mu_{1} \varrho_{0}^{2}\right)\right] l(l+1)$ und sind damit normale Rotationsbanden.

Unser Beispiel zeigt also den Übergang von der völlig entkoppelten Bewegung, die durch $l_{1}$ und $l_{2}$ beschrieben wird, bis zur stark gekoppelten, die durch $l, n$ und den Zustand von $\mu_{2} r_{0}^{2}$ charakterisiert ist und bei welcher $\mu_{2}$ der "langsamen Rotation“ von $\mu_{1}$ wegen des $u$-abhängigen Potentials adiabatisch folgt.

$$
\text { II. } \varrho \text { starr, } V=V(r u)
$$

Dieser Fall entspricht den Modellen für u-g-Kerne: Der aus den Massen $m_{1}$ und $m_{2}$ gebildete Rumpf besitzt um die Verbindungslinie $\varrho$ kein Trägheitsmoment, und damit ist auch der Drehimpuls des Rumpfes um diese Achse immer Null, wie es experimentell beobachtet wird. Da in der Nebendiagonale bei $\varrho$-Richtung-Quantisierung kein $r$ vorkommt, ändert sich die Struktur der Matrizen nicht. Lediglich die Elemente der Untermatrizen werden jetzt ebenfalls Matrizen, deren Länge durch die Anzahl der Entwicklungsfunktionen für die $r$-Abhängigkeit gegeben ist, und die Nebendiagonalen werden entspre- chend länger. Im übrigen treffen alle unter I angestellten Überlegungen auch hier zu. Ein qualitativer Vergleich mit den vorhandenen Modellen ist nur möglich, wenn man den Spin von Teilchen 3 mitnimmt. Dies geschieht unter Verwendung der hier gewonnenen Erfahrungen bei NäPfEL ${ }^{10}$. Wir beschränken uns deshalb im folgenden auf atomare Systeme mit Coulomb-Wechselwirkung, bei denen der Spin keine wesentliche Rolle spielt.

$$
\text { III. } V=V(\varrho r u)
$$

Unsere vorhergehenden Überlegungen sind methodisch zugeschnitten auf solche 3-Teilchen-Systeme, die genau 2 gleiche Teilchen enthalten. Im atomaren Bereich gehören hierzu das Heliumatom und heliumähnliche Ionen sowie das $\mathrm{H}_{2}^{+}$-Ion und ähnliche $\mathrm{Ge}$ bilde. Die Ortsanteile der Wellenfunktionen solcher Systeme verhalten sich bei Teilchenvertauschung symmetrisch oder antisymmetrisch. Im Prinzip ist es möglich, alle Eigenzustände zu berechnen, indem wir die $u$-Abhängigkeit wie oben nach $Y_{l_{2} n}(u, 0)$ und die $\varrho$ - und $r$-Abhängigkeit nach geeigneten Polynomen entwickeln und damit die nach Gesamtdrehimpuls und Parität getrennten Differentialgleichungssysteme lösen. Die für die Matrixelemente benötigten Integrale lassen sich geschlossen auswerten, wenn man die in der potentiellen Energie vorkommenden Abstände zwischen Teilchen 1 und 3 sowie Teilchen 2 und 3 folgendermaßen entwickelt (Teilchen 1 und 2 sollen wieder gleich sein) :

$$
\frac{1}{\sqrt{1} \varrho^{2}+r^{2}+\varrho r \cos u}+\frac{1}{\sqrt{\frac{1}{4} \varrho^{2}+r^{2}-\varrho r \cos u}}= \begin{cases}\frac{4}{\varrho} \sum_{\nu=0}^{\infty}\left(\frac{2 r}{\varrho}\right)^{2 v} P_{2 \nu}(\cos u) \text { für } \quad r \leqq \varrho / 2, \\ \frac{2}{r} \sum_{\nu=0}^{\infty}\left(\frac{\varrho}{2 r}\right)^{2 v} P_{2 \nu}(\cos u) \text { für } \quad \varrho / 2 \leqq r .\end{cases}
$$

Während jedoch die Entwicklung der Eigenfunktionen nach $Y_{l_{2} n}(u, 0)$ bei unserem Beispiel in I und bei den in II erwähnten Kernmodellen gut konvergiert, ist dies bei der Coulomb-Wechselwirkung nicht mehr der Fall. Das hat folgenden physikalischen Grund: Bei den zuerst genannten Problemen ist die potentielle Energie eine relativ glatte Funktion von $u$. Bei der Coulomb-Wechselwirkung dagegen enthält sie für $r=\varrho / 2$ an den Stellen $u=0$ und $u=\pi$ Singularitäten. Wenn zwischen Teilchen 1 und 3 sowie Teilchen 2 und 3 Anziehung herrscht, ist die Wellenfunktion in diesem Bereich besonders groß und besitzt dort eine Spitze. Um diese Spitze in der $u$-Abhängigkeit mit $Y_{l_{2} n}(u, 0)$ gut anzunähern, müssen wir viele Funktionen mitnehmen. Da wir das Verhalten der exakten Lösung an den singulären Punkten kennen, gibt uns diese Utberlegung gleichzeitig einen Hinweis, wie wir eine bessere Konvergenz erzielen können. Wir ziehen dazu eine geeignet gewählte Funktion als Faktor heraus und entwickeln den "glatteren“ Rest. Dabei geht zwar die Orthogonalität unserer Entwicklungsfunktionen verloren, aber das ist für das Diagonalisierungsverfahren belanglos. 
Mit dieser Methode behandeln wir das Heliumatom. Die Ordnungszahl $Z$ lassen wir dabei zunächst allgemein stehen. Es ist dann

Weiter führen wir dimensionslose Größen ein:

$$
m_{1}=m_{2}=m_{\mathrm{e}}, \quad m_{3}=m_{\mathrm{He}}, \quad \mu_{1}=m_{\mathrm{e}} / 2, \quad \mu_{2}=\frac{2 m_{\mathrm{e}}}{1+2 m_{\mathrm{e}} / m_{\mathrm{He}}} .
$$

$$
\varrho^{\prime}=\frac{1}{2} \frac{Z e^{2} 2 m_{\mathrm{e}}}{4 \pi \varepsilon_{0} \hbar^{2}} \varrho, \quad r^{\prime}=\frac{Z e^{2} 2 m_{\mathrm{e}}}{4 \pi \varepsilon_{0} \hbar^{2}} r, \quad \lambda=\frac{\left(4 \pi \varepsilon_{0}\right)^{2} \hbar^{2}}{Z^{2} e^{4} m_{\mathrm{e}}} E .
$$

Für $l=0$ erhalten wir die folgende Eigenwertgleichung:

$$
\begin{gathered}
\left\{\left[\frac{\partial^{2}}{\partial \varrho^{\prime 2}}+\frac{2}{\varrho^{\prime}} \frac{\partial}{\partial \varrho^{\prime}}+\frac{1}{\varrho^{\prime 2}}\left(\frac{\partial^{2}}{\partial u^{2}}+\cot u \frac{\partial}{\partial u}\right)\right]+\left(1+\frac{2 m_{\mathrm{e}}}{m_{\mathrm{He}}}\right)\left[\frac{\partial^{2}}{\partial r^{\prime 2}}+\frac{2}{r^{\prime}} \frac{\partial}{\partial r^{\prime}}+\frac{1}{r^{\prime 2}}\left(\frac{\partial^{2}}{\partial u^{2}}+\cot u \frac{\partial}{\partial u}\right)\right]\right. \\
\left.+2\left[\frac{1}{\sqrt{\varrho^{\prime 2}+r^{\prime 2}-2 \varrho^{\prime} r^{\prime} \cos u}}+\frac{1}{\sqrt{\varrho^{\prime 2}+r^{\prime 2}+2 \varrho^{\prime} r^{\prime} \cos u}}-\frac{1}{2 Z \varrho^{\prime}}\right]+\lambda\right\} \Psi=0 .
\end{gathered}
$$

Den He-Kern nehmen wir als unendlich schwer an, bemerken aber, daß dabei der Charakter als 3-KörperProblem keineswegs verloren geht und daß auch eine endliche Masse $m_{3}$ in unseren Koordinaten relativ einfach mitzunehmen wäre. Für die Wellenfunktion machen wir auf Grund unserer obigen Überlegungen den Ansatz:

$$
\begin{aligned}
& \Psi=\Psi_{0}\left(\varrho^{\prime} r^{\prime} u\right) f\left(\varrho^{\prime} r^{\prime} u\right) \operatorname{mit} \\
& \qquad \Psi_{0}=\exp \left\{-\frac{1}{2} \sqrt{\varrho^{\prime 2}+r^{\prime 2}-2 \varrho^{\prime} r^{\prime} \cos u}-\frac{1}{2} \sqrt{\varrho^{\prime 2}+r^{\prime 2}+2 \varrho^{\prime} r^{\prime} \cos u}+\frac{1}{2 Z} \varrho^{\prime}\right\}
\end{aligned}
$$

der, in die Eigenwertgleichung eingesetzt, liefert:

$$
\begin{gathered}
\Psi_{0}\left[\frac{\partial^{2}}{\partial \varrho^{\prime 2}}+\frac{2}{\varrho^{\prime}} \frac{\partial}{\partial \varrho^{\prime}}+\frac{1}{\varrho^{\prime 2}}\left(\frac{\partial^{2}}{\partial u^{2}}+\cot u \frac{\partial}{\partial u}\right)+\frac{\partial^{2}}{\partial r^{\prime 2}}+\frac{2}{r^{\prime}} \frac{\partial}{\partial r^{\prime}}+\frac{1}{r^{\prime 2}}\left(\frac{\partial^{2}}{\partial u^{2}}+\cot u \frac{\partial}{\partial u}\right)+\left(1-\frac{1}{(2 Z)^{2}}+\lambda\right)\right] f \\
+2\left[\frac{\partial \Psi_{0}}{\partial \varrho^{\prime}}\left(\frac{1}{2 Z}+\frac{\partial}{\partial \varrho^{\prime}}\right)+\frac{\partial \Psi_{0}^{\prime}}{\partial r^{\prime}} \frac{\partial}{\partial r^{\prime}}+\left(\frac{1}{\varrho^{\prime 2}}+\frac{1}{r^{\prime 2}}\right) \frac{\partial \Psi_{0}}{\partial u} \frac{\partial}{\partial u}\right] f=0 .
\end{gathered}
$$

Da $\Psi_{0}\left(\varrho^{\prime} r^{\prime} u\right)=\Psi_{0}\left(\varrho^{\prime} r^{\prime} \pi-u\right)$ ist, also symmetrisch bezüglich Teilchenvertauschung, hat $\Psi$ die gleiche Symmetrie wie $f$. Für den symmetrischen Grundzustand sieht daher die Entwicklung von $f$ folgendermaßen aus:

$$
\begin{aligned}
& f\left(\varrho^{\prime} r^{\prime} u\right) \\
& =\left\{g_{0}\left(\varrho^{\prime} r^{\prime}\right) Y_{00}(u, 0)+g_{2}\left(\varrho^{\prime} r^{\prime}\right) Y_{20}(u, 0)+\ldots\right\} .
\end{aligned}
$$

Wir haben drei Näherungen gerechnet, an denen man die gute Konvergenz des Verfahrens erkennt.

$$
\begin{array}{rlrl}
f & =1: & E & =-2,85550 \text { atom. Einh. } \\
f & =1+c_{1} \varrho^{\prime}: & E & =-2,87919 \text { atom. Einh. } \\
f & =1+c_{1} \varrho^{\prime}+c_{2} r^{\prime 2}: E & =-2,90001 \text { atom. Einh. }
\end{array}
$$

Ein Vergleich mit dem genaueren Wert

$$
E=-2,90372 \text { a. E. }
$$

zeigt, daß unser Ergebnis, das nur 2 Parameter enthält, auf ca. $1 \%$ genau ist. Wir haben damit auch für Systeme mit Coulomb-Wechselwirkung eine Methode gefunden, die mit übersichtlichen Entwicklungsfunktionen rasch konvergiert.

Wir stellen noch für $Z \geqq 2$ unseren Ansatz nullter Näherung für den Grundzustand $(f=1)$ dem für Systeme dieser Art üblichen einparametrigen Varia- tionsansatz

$$
\begin{aligned}
\exp \left\{-k\left(\sqrt{\varrho^{\prime 2}}+r^{\prime 2}-2 \varrho^{\prime} r^{\prime} \cos u\right.\right. \\
\left.\left.+\sqrt{\varrho^{\prime 2}+r^{\prime 2}+2 \varrho^{\prime} r^{\prime} \cos u}\right)\right\}
\end{aligned}
$$

in Tab. 1 gegenüber.

\begin{tabular}{cccc}
\hline$Z$ & Variation & $f=1$ & Experiment \\
\hline 2 & $-2,848$ & $-2,856$ & $-2,904$ \\
3 & $-7,223$ & $-7,235$ & $-7,280$ \\
4 & $-13,598$ & $-13,612$ & $-13,656$ \\
5 & $-21,973$ & $-21,988$ & $-22,032$ \\
\hline
\end{tabular}

Tab. 1. Berechnete und gemessene Grundzustandsenergien in atomaren Einheiten für verschiedene Ordnungszahlen $Z$.

Unser vor die Lösungsentwicklung gezogener Faktor $\Psi_{0}\left(\varrho^{\prime} r^{\prime} u\right)$ eignet sich also für jedes $Z \geqq 2$ und ergibt stets einen besseren Wert als der einparametrige Variationsansatz, wobei die Güte mit wachsendem $Z$ noch zunimmt.

Herr Professor Dr. H. Volz widmete dieser Arbeit viel Zeit und Interesse und gab mir in zahlreichen Diskussionen viele wertvolle Anregungen. Ich möchte mich dafür herzlich bedanken. - Der freundschaftliche Gedankenaustausch mit Herrn H. NäPfEL hat zur Klärung mancher Probleme beigetragen. - Der Firma Si e mens A.G. danke ich für die großzügig zur Verfügung gestellte Rechenzeit. 